\title{
A FRAMEWORK OF ROGERS-RAMANUJAN IDENTITIES AND THEIR ARITHMETIC PROPERTIES
}

\author{
MICHAEL J. GRIFFIN, KEN ONO, AND S. OLE WARNAAR \\ In memory of Basil Gordon and Alain Lascoux
}

Abstract. The two Rogers-Ramanujan $q$-series

$$
\sum_{n=0}^{\infty} \frac{q^{n(n+\sigma)}}{(1-q) \cdots\left(1-q^{n}\right)}
$$

where $\sigma=0,1$, play many roles in mathematics and physics. By the Rogers-Ramanujan identities, they are essentially modular functions. Their quotient, the Rogers-Ramanujan continued fraction, has the special property that its singular values are algebraic integral units. We find a framework which extends the Rogers-Ramanujan identities to doublyinfinite families of $q$-series identities. If $a \in\{1,2\}$ and $m, n \geq 1$, then we have

$$
\sum_{\substack{\lambda \\ \lambda_{1} \leq m}} q^{a|\lambda|} P_{2 \lambda}\left(1, q, q^{2}, \ldots ; q^{n}\right)=\text { "infinite product modular function", }
$$

where the $P_{\lambda}\left(x_{1}, x_{2}, \ldots ; q\right)$ are Hall-Littlewood polynomials. These $q$-series are specialized characters of affine Kac-Moody algebras. Generalizing the Rogers-Ramanujan continued fraction, we prove in the case of $\mathrm{A}_{2 n}^{(2)}$ that the relevant $q$-series quotients are integral units.

\section{INTRODUCTION}

The Rogers-Ramanujan (RR) identities 69.

$$
G(q):=\sum_{n=0}^{\infty} \frac{q^{n^{2}}}{(1-q) \cdots\left(1-q^{n}\right)}=\prod_{n=0}^{\infty} \frac{1}{\left(1-q^{5 n+1}\right)\left(1-q^{5 n+4}\right)}
$$

and

$$
H(q):=\sum_{n=0}^{\infty} \frac{q^{n^{2}+n}}{(1-q) \cdots\left(1-q^{n}\right)}=\prod_{n=0}^{\infty} \frac{1}{\left(1-q^{5 n+2}\right)\left(1-q^{5 n+3}\right)}
$$

2010 Mathematics Subject Classification. 05E05, 05E10, 11P84, 11G16, 17B67, 33D67.

The first two authors are supported by the NSF, and the third author is supported by the Australian Research Council. The second author also thanks the support of the Asa Griggs Candler Fund. 
play many roles in mathematics and physics. They are essentially modular functions, and their ratio $H(q) / G(q)$ is the famous Rogers-Ramanujan $q$-continued fraction

$$
\frac{H(q)}{G(q)}=\frac{1}{1+\frac{q}{1+\frac{q^{2}}{1+\frac{q^{3}}{\ddots}}} .}
$$

The golden ratio $\phi$ satisfies $H(1) / G(1)=1 / \phi=(-1+\sqrt{5}) / 2$. Ramanujan computed further values such as 1

$$
\mathrm{e}^{-\frac{2 \pi}{5}} \cdot \frac{H\left(\mathrm{e}^{-2 \pi}\right)}{G\left(\mathrm{e}^{-2 \pi}\right)}=\sqrt{\frac{5+\sqrt{5}}{2}}-\frac{\sqrt{5}+1}{2} .
$$

The minimal polynomial of this value is

$$
x^{4}+2 x^{3}-6 x^{2}-2 x+1
$$

which shows that it is an algebraic integral unit. All of Ramanujan's evaluations are such units.

Ramanujan's evaluations inspired early work by Watson [60, 78, 79] and Ramanathan [67]. Then in 1996, Berndt, Chan and Zhang [11] finally obtained general theorems concerning such values. The theory pertains to values at $q:=\mathrm{e}^{2 \pi \mathrm{i} \tau}$, where the $\tau$ are quadratic irrational points in the upper-half of the complex plane. We refer to such a point $\tau$ as a $C M$ point with discriminant $-D<0$, where $-D$ is the discriminant of the minimal polynomial of $\tau$. The corresponding evaluation is known as a singular value. Berndt, Chan and Zhang proved that the singular values $q^{-1 / 60} G(q)$ and $q^{11 / 60} H(q)$ are algebraic numbers in abelian extensions of $\mathbb{Q}(\tau)$ which satisfy the exceptional property (see [11, Theorem 6.2]) that their ratio $q^{1 / 5} H(q) / G(q)$ is an algebraic integral unit which generates specific abelian extensions of $\mathbb{Q}(\tau)$.

Remark. The individual values of $q^{-1 / 60} G(q)$ and $q^{11 / 60} H(q)$ generically are not algebraic integers. For example, in (1.4) we have $\tau=\mathrm{i}$, and the numerator and denominator

$$
q^{-\frac{1}{60}} G(q)=\sqrt[4]{\frac{1+3 \sqrt{5}+2 \sqrt{10+2 \sqrt{5}}}{10}} \text { and } q^{\frac{11}{60} H(q)}=\sqrt[4]{\frac{1+3 \sqrt{5}-2 \sqrt{10+2 \sqrt{5}}}{10}}
$$

share the minimal polynomial $625 x^{16}-250 x^{12}-1025 x^{8}-90 x^{4}+1$.

In addition to the algebraic properties described above, (1.1) and (1.2) have been related to a large number of different areas of mathematics. They were were first recognized by MacMahon and Schur as identities for integer partitions [59, 71], but have since been

\footnotetext{
${ }^{1} \mathrm{He}$ offered this value in his first letter to Hardy (see p. 29 of [12]).

${ }^{2}$ Cais and Conrad [24] and Duke [27] later revisited these results from the perspective of arithmetic geometry and the symmetries of the regular icosahedron respectively.
} 
linked to algebraic geometry [23, 37], K-theory [28], conformal field theory [10, 44, 52], group theory [32], Kac-Moody, Virasoro, vertex and double affine Hecke algebras [25, 31, 50, 51, 53, 54, 55, 56], knot theory [6, 39, 40], modular forms [16, 17, 18, 19, 20, 21, 22, 63], orthogonal polynomials [7, 15, 35], statistical mechanics [4, 9], probability [33] and transcendental number theory 68.

In 1974 Andrews [1] extended (1.1) and (1.2) to an infinite family of Rogers-Ramanujan-type identities by proving that

$$
\sum_{r_{1} \geq \cdots \geq r_{m} \geq 0} \frac{q^{r_{1}^{2}+\cdots+r_{m}^{2}+r_{i}+\cdots+r_{m}}}{(q)_{r_{1}-r_{2}} \cdots(q)_{r_{m-1}-r_{m}}(q)_{r_{m}}}=\frac{\left(q^{2 m+3} ; q^{2 m+3}\right)_{\infty}}{(q)_{\infty}} \cdot \theta\left(q^{i} ; q^{2 m+3}\right),
$$

where $1 \leq i \leq m+1$. As usual, here we have that

$$
(a)_{k}=(a ; q)_{k}:= \begin{cases}(1-a)(1-a q) \cdots\left(1-a q^{k-1}\right) & \text { if } k \geq 0, \\ \prod_{j=0}^{\infty}\left(1-a q^{j}\right) & \text { if } k=\infty\end{cases}
$$

and

$$
\theta(a ; q):=(a ; q)_{\infty}(q / a ; q)_{\infty}
$$

is a modified theta function. The identities (1.5), which can be viewed as the analytic counterpart of Gordon's partition theorem [36], are now commonly referred to as the Andrews-Gordon (AG) identities.

Remark. The specializations of $\theta(a ; q)$ in (1.5) are (up to powers of $q$ ) modular functions, where $q:=\mathrm{e}^{2 \pi \mathrm{i} \tau}$ and $\tau$ is any complex point with $\operatorname{Im}(\tau)>0$. It should be noted that this differs from our use of $q$ and $\tau$ above where we required $\tau$ to be a quadratic irrational point. Such infinite product modular functions were studied extensively by Klein and Siegel.

There are numerous algebraic interpretations of the Rogers-Ramanujan and AndrewsGordon identities. For example, the above-cited papers by Milne, Lepowsky and Wilson show that they arise, up to a factor $\left(q ; q^{2}\right)_{\infty}$, as principally specialized characters of integrable highest-weight modules of the affine Kac-Moody algebra $\mathrm{A}_{1}^{(1)}$. Similarly, Feigin and Frenkel proved the Rogers-Ramanujan and Andrews-Gordon identities by considering certain irreducible minimal representations of the Virasoro algebra [31]. We should also mention the much larger program by Lepowsky and others on combinatorial and algebraic extensions of Rogers-Ramanujan-type identities, leading to the introduction of $Z$-algebras for all affine Lie algebras, vertex-operator-theoretic proofs of generalized Rogers-Ramanujan identities, and Rogers-Ramanujan-type identities for arbitrary affine Lie algebras in which, typically, the sum side is replaced by a combinatorial sum, see e.g., [34, 49, 62 and references therein.

In this paper we have a similar but distinct aim, namely to find a concrete framework of Rogers-Ramanujan type identities in the $q$-series sense of "infinite sum = infinite product", where the infinite products arise as specialized characters of appropriately chosen affine 
Lie algebras $\mathrm{X}_{N}^{(r)}$ for arbitrary $N$. Such a general framework would give new connections between Lie algebras and the theory of modular functions.

In [5] (see also [30, 76]) some results concerning the above question were obtained, resulting in Rogers-Ramanujan-type identities for $\mathrm{A}_{2}^{(1)}$. The approach of [5] does not in any obvious manner extend to $\mathrm{A}_{n}^{(1)}$ for all $n$, and this paper aims to give a more complete answer. By using a level- $m$ Rogers-Selberg identity for the root system $\mathrm{C}_{n}$ as recently obtain by Bartlett and the third author [8], we show that the Rogers-Ramanujan and Andrews-Gordon identities are special cases of a doubly-infinite family of $q$-identities arising from the Kac-Moody algebra $\mathrm{A}_{2 n}^{(2)}$ for arbitrary $n$. In their most compact form, the "sum-sides" are expressed in terms of Hall-Littlewood polynomials $P_{\lambda}(x ; q)$ evaluated at infinite geometric progressions (see Section 2 for definitions and further details), and the "product-sides" are essentially products of modular theta functions. We shall present four pairs $(a, b)$ such that for all $m, n \geq 1$ we have an identity of the form

$$
\sum_{\substack{\lambda \\ \lambda_{1} \leq m}} q^{a|\lambda|} P_{2 \lambda}\left(1, q, q^{2}, \ldots ; q^{2 n+b}\right)=\text { "infinite product modular function". }
$$

To make this precise, we fix notation for integer partitions, nonincreasing sequences of nonnegative integers with at most finitely many nonzero terms. For a partition $\lambda=$ $\left(\lambda_{1}, \lambda_{2}, \ldots\right)$, we let $|\lambda|:=\lambda_{1}+\lambda_{2}+\cdots$, and we let $2 \lambda:=\left(2 \lambda_{1}, 2 \lambda_{2}, \ldots\right)$. We also require $\lambda^{\prime}$, the conjugate of $\lambda$, the partition which is obtained by transposing the Ferrers-Young diagram of $\lambda$. Finally, for convenience we let

$$
\theta\left(a_{1}, \ldots, a_{k} ; q\right):=\theta\left(a_{1} ; q\right) \cdots \theta\left(a_{k} ; q\right)
$$

Example. If $\lambda=(5,3,3,1)$, then we have that $|\lambda|=12,2 \lambda=(10,6,6,2)$ and $\lambda^{\prime}=$ $(4,3,3,1,1)$.

Using this notation, we have the following pair of doubly-infinite Rogers-Ramanujan type identities which correspond to specialized characters of $\mathrm{A}_{2 n}^{(2)}$.

Theorem $1.1\left(\mathrm{~A}_{2 n}^{(2)} \mathrm{RR}\right.$ and $\mathrm{AG}$ identities). If $m$ and $n$ are positive integers and $\kappa:=$ $2 m+2 n+1$, then we have that

$$
\begin{aligned}
\sum_{\substack{\lambda \\
\lambda_{1} \leq m}} q^{|\lambda|} & P_{2 \lambda}\left(1, q, q^{2}, \ldots ; q^{2 n-1}\right) \\
& =\frac{\left(q^{\kappa} ; q^{\kappa}\right)_{\infty}^{n}}{(q)_{\infty}^{n}} \cdot \prod_{i=1}^{n} \theta\left(q^{i+m} ; q^{\kappa}\right) \prod_{1 \leq i<j \leq n} \theta\left(q^{j-i}, q^{i+j-1} ; q^{\kappa}\right) \\
& =\frac{\left(q^{\kappa} ; q^{\kappa}\right)_{\infty}^{m}}{(q)_{\infty}^{m}} \cdot \prod_{i=1}^{m} \theta\left(q^{i+1} ; q^{\kappa}\right) \prod_{1 \leq i<j \leq m} \theta\left(q^{j-i}, q^{i+j+1} ; q^{\kappa}\right)
\end{aligned}
$$


and

$$
\begin{aligned}
\sum_{\substack{\lambda \\
\lambda_{1} \leq m}} q^{2|\lambda|} & P_{2 \lambda}\left(1, q, q^{2}, \ldots ; q^{2 n-1}\right) \\
= & \frac{\left(q^{\kappa} ; q^{\kappa}\right)_{\infty}^{n}}{(q)_{\infty}^{n}} \cdot \prod_{i=1}^{n} \theta\left(q^{i} ; q^{\kappa}\right) \prod_{1 \leq i<j \leq n} \theta\left(q^{j-i}, q^{i+j} ; q^{\kappa}\right) \\
= & \frac{\left(q^{\kappa} ; q^{\kappa}\right)_{\infty}^{m}}{(q)_{\infty}^{m}} \cdot \prod_{i=1}^{m} \theta\left(q^{i} ; q^{\kappa}\right) \prod_{1 \leq i<j \leq m} \theta\left(q^{j-i}, q^{i+j} ; q^{\kappa}\right) .
\end{aligned}
$$

Remarks. (1) When $m=n=1$, Theorem 1.1 gives the Rogers-Ramanujan identities (1.1) and (1.2). The summation defining the series is over the empty partition, $\lambda=0$, and partitions consisting of $n$ copies of 1 , i.e., $\lambda=\left(1^{n}\right)$. Since

$$
q^{(\sigma+1)\left|\left(1^{n}\right)\right|} P_{\left(2^{n}\right)}\left(1, q, q^{2}, \ldots ; q\right)=\frac{q^{n(n+\sigma)}}{(1-q) \cdots\left(1-q^{n}\right)},
$$

identities (1.1) and (1.2) thus follow from Theorem 1.1 by letting $\sigma=0,1$.

(2) When $n=1$, Theorem 1.1 gives the $i=1$ and the $i=m+1$ instances of the AndrewsGordon identities in a representation due to Stembridge [74] (see also Fulman [32]). The equivalence with (1.5) follows from the specialization formula [58, p. 213]

$$
q^{(\sigma+1)|\lambda|} P_{2 \lambda}\left(1, q, q^{2}, \ldots ; q\right)=\prod_{i \geq 1} \frac{q^{r_{i}\left(r_{i}+\sigma\right)}}{(q)_{r_{i}-r_{i+1}}},
$$

where $r_{i}:=\lambda_{i}^{\prime}$. Note that $\lambda_{1} \leq m$ implies that $\lambda_{i}^{\prime}=r_{i}=0$ for $i>m$.

(3) We note the beautiful level-rank duality exhibited by the products on the right-hand sides of the expressions in Theorem 1.1 (especially those of (1.7b)).

(4) In the next section we shall show that the more general series

$$
\sum_{\substack{\lambda \\ \lambda_{1} \leq m}} q^{(\sigma+1)|\lambda|} P_{2 \lambda}\left(1, q, q^{2}, \ldots ; q^{n}\right)
$$

are also expressible in terms of $q$-shifted factorials, allowing for a formulation of Theorem 1.1 (see Lemma 2.11) which is independent of Hall-Littlewood polynomials.

Example. Here we illustrate Theorem 1.1 when $m=n=2$. Then (1.7a) is

$$
\sum_{\substack{\lambda \\ \lambda_{1} \leq 2}} q^{|\lambda|} P_{2 \lambda}\left(1, q, q^{2}, \ldots ; q^{3}\right)=\prod_{n=1}^{\infty} \frac{\left(1-q^{9 n}\right)}{\left(1-q^{n}\right)}
$$

giving another expression for the $q$-series studied by Dyson in his "A walk through Ramanujan's Garden" [29]: 
"The end of the war was not in sight. In the evenings of that winter I kept sane by wandering in Ramanujan's garden. ... I found a lot of identities of the sort that Ramanujan would have enjoyed. My favorite one was this one:

$$
\sum_{n=0}^{\infty} x^{n^{2}+n} \cdot \frac{\left(1+x+x^{2}\right)\left(1+x^{2}+x^{4}\right) \cdots\left(1+x^{n}+x^{2 n}\right)}{(1-x)\left(1-x^{2}\right) \cdots\left(1-x^{2 n+1}\right)}=\prod_{n=1}^{\infty} \frac{\left(1-x^{9 n}\right)}{\left(1-x^{n}\right)} .
$$

In the cold dark evenings, while I was scribbling these beautiful identities amid the death and destruction of 1944, I felt close to Ramanujan. He had been scribbling even more beautiful identities amid the death and destruction of 1917."

The series in (1.7b) is

$$
\sum_{\substack{\lambda \\ \lambda_{1} \leq 2}} q^{2|\lambda|} P_{2 \lambda}\left(1, q, q^{2}, \ldots ; q^{3}\right)=\prod_{n=1}^{\infty} \frac{\left(1-q^{9 n}\right)\left(1-q^{9 n-1}\right)\left(1-q^{9 n-8}\right)}{\left(1-q^{n}\right)\left(1-q^{9 n-4}\right)\left(1-q^{9 n-5}\right)} .
$$

We also have an even modulus analog of Theorem 1.1. Surprisingly, the $a=1$ and $a=2$ cases correspond to dual affine Lie algebras, namely $\mathrm{C}_{n}^{(1)}$ and $\mathrm{D}_{n+1}^{(2)}$.

Theorem $1.2\left(\mathrm{C}_{n}^{(1)} \mathrm{RR}\right.$ and $\mathrm{AG}$ identities). If $m$ and $n$ are positive integers and $\kappa:=$ $2 m+2 n+2$, then we have that

$$
\begin{aligned}
\sum_{\substack{\lambda \\
\lambda_{1} \leq m}} q^{|\lambda|} & P_{2 \lambda}\left(1, q, q^{2}, \ldots ; q^{2 n}\right) \\
& =\frac{\left(q^{2} ; q^{2}\right)_{\infty}\left(q^{\kappa / 2} ; q^{\kappa / 2}\right)_{\infty}\left(q^{\kappa} ; q^{\kappa}\right)_{\infty}^{n-1}}{(q)_{\infty}^{n+1}} \cdot \prod_{i=1}^{n} \theta\left(q^{i} ; q^{\kappa / 2}\right) \prod_{1 \leq i<j \leq n} \theta\left(q^{j-i}, q^{i+j} ; q^{\kappa}\right) \\
& =\frac{\left(q^{\kappa} ; q^{\kappa}\right)_{\infty}^{m}}{(q)_{\infty}^{m}} \cdot \prod_{i=1}^{m} \theta\left(q^{i+1} ; q^{\kappa}\right) \prod_{1 \leq i<j \leq m} \theta\left(q^{j-i}, q^{i+j+1} ; q^{\kappa}\right) .
\end{aligned}
$$

Theorem $1.3\left(\mathrm{D}_{n+1}^{(2)} \mathrm{RR}\right.$ and $\mathrm{AG}$ identities). If $m$ and $n$ are positive integers such that $n \geq 2$, and $\kappa:=2 m+2 n$, then we have that

$$
\begin{aligned}
\sum_{\substack{\lambda \\
\lambda_{1} \leq m}} q^{2|\lambda|} & P_{2 \lambda}\left(1, q, q^{2}, \ldots ; q^{2 n-2}\right) \\
& =\frac{\left(q^{\kappa} ; q^{\kappa}\right)_{\infty}^{n}}{\left(q^{2} ; q^{2}\right)_{\infty}(q)_{\infty}^{n-1}} \cdot \prod_{1 \leq i<j \leq n} \theta\left(q^{j-i}, q^{i+j-1} ; q^{\kappa}\right) \\
& =\frac{\left(q^{\kappa} ; q^{\kappa}\right)_{\infty}^{m}}{(q)_{\infty}^{m}} \cdot \prod_{i=1}^{m} \theta\left(q^{i} ; q^{\kappa}\right) \prod_{1 \leq i<j \leq m} \theta\left(q^{j-i}, q^{i+j} ; q^{\kappa}\right) .
\end{aligned}
$$


Remarks. (1) The $(m, n)=(1,2)$ case of (1.10) is equivalent to Milne's modulus 6 RogersRamanujan identity [61, Theorem 3.26].

(2) If we take $m=1$ in (1.9) (with $n \mapsto n-1$ ) and (1.10), and apply formula (2.7) below (with $\delta=0$ ), we obtain the $i=1,2$ cases of Bressoud's even modulus identities [13]

$$
\sum_{r_{1} \geq \cdots \geq r_{n} \geq 0} \frac{q^{r_{1}^{2}+\cdots+r_{n}^{2}+r_{i}+\cdots+r_{n}}}{(q)_{r_{1}-r_{2}} \cdots(q)_{r_{n-1}-r_{n}}\left(q^{2} ; q^{2}\right)_{r_{n}}}=\frac{\left(q^{2 n+2} ; q^{2 n+2}\right)_{\infty}}{(q)_{\infty}} \cdot \theta\left(q^{i} ; q^{2 n+2}\right) .
$$

By combining (1.7)-(1.10), we obtain an identity of "mixed" type.

Corollary 1.4. If $m$ and $n$ are positive integers and $\kappa:=2 m+n+2$, then for $\sigma=0,1$ we have that

$$
\begin{aligned}
& \sum_{\substack{\lambda \\
\lambda_{1} \leq m}} q^{(\sigma+1)|\lambda|} P_{2 \lambda}\left(1, q, q^{2}, \ldots ; q^{n}\right) \\
& \quad=\frac{\left(q^{\kappa} ; q^{\kappa}\right)_{\infty}^{m}}{(q)_{\infty}^{m}} \cdot \prod_{i=1}^{m} \theta\left(q^{i-\sigma+1} ; q^{\kappa}\right) \prod_{1 \leq i<j \leq m} \theta\left(q^{j-i}, q^{i+j-\sigma+1} ; q^{\kappa}\right) .
\end{aligned}
$$

Identities for $\mathrm{A}_{n-1}^{(1)}$ also exist, although their formulation is perhaps slightly less satisfactory. We have the following "limiting" Rogers-Ramanujan type identities.

Theorem $1.5\left(\mathrm{~A}_{n-1}^{(1)} \mathrm{RR}\right.$ and $\mathrm{AG}$ identities). If $m$ and $n$ are positive integers and $\kappa:=$ $m+n$, then we have that

$$
\begin{aligned}
& \lim _{r \rightarrow \infty} q^{-m\left(\begin{array}{c}
r \\
2
\end{array}\right)} P_{\left(m^{r}\right)}\left(1, q, q^{2}, \ldots ; q^{n}\right)=\frac{\left(q^{\kappa} ; q^{\kappa}\right)_{\infty}^{n-1}}{(q)_{\infty}^{n}} \cdot \prod_{1 \leq i<j \leq n} \theta\left(q^{j-i} ; q^{\kappa}\right) \\
& =\frac{\left(q^{\kappa} ; q^{\kappa}\right)_{\infty}^{m-1}}{(q)_{\infty}^{m}} \cdot \prod_{1 \leq i<j \leq m} \theta\left(q^{j-i} ; q^{\kappa}\right) \text {. }
\end{aligned}
$$

Now we turn to the question of whether the new $q$-series appearing in these theorems, which arise from the Hall-Littlewood polynomials, enjoy the same algebraic properties as (1.1), (1.2), and the Rogers-Ramanujan continued fraction. As it turns out they do: their singular values are algebraic numbers. Moreover, we characterize those ratios which simplify to algebraic integral units.

To make this precise, we recall that $q=\mathrm{e}^{2 \pi \mathrm{i} \tau}$ for $\operatorname{Im}(\tau)>0$, and that $m$ and $n$ are arbitrary positive integers. The auxiliary parameter $\kappa=\kappa_{*}(m, n)$ in Theorems 1.1, 1.2 and 1.3 is defined as follows:

$$
\kappa= \begin{cases}\kappa_{1}(m, n):=2 m+2 n+1 & \text { for } \mathrm{A}_{2 n}^{(2)} \\ \kappa_{2}(m, n):=2 m+2 n+2 & \text { for } \mathrm{C}_{n}^{(1)} \\ \kappa_{3}(m, n):=2 m+2 n & \text { for } \mathrm{D}_{n+1}^{(2)}\end{cases}
$$


Remark. The parameter $\kappa$ has a representation theoretic interpretation arising from the corresponding affine Lie algebra $X_{N}^{(r)}$ (see Section 3). It turns out that

$$
\kappa_{*}(m, n)=\frac{2}{r}\left(\operatorname{lev}(\Lambda)+h^{\vee}\right)
$$

where $\operatorname{lev}(\Lambda)$ is the level of the corresponding representation, $h^{\vee}$ is the dual Coxeter number and $r$ is the tier number.

To obtain algebraic values, we require certain normalizations of these series. The subscripts below correspond to the labelling in the theorems. In particular, $\Phi_{1 a}$ and $\Phi_{1 b}$ appear in Theorem 1.1, $\Phi_{2}$ is in Theorem 1.2, and $\Phi_{3}$ is in Theorem 1.3. Using this notation, the series are

$$
\begin{aligned}
\Phi_{1 a}(m, n ; \tau): & =q^{\frac{m n(4 m n-4 m+2 n-3)}{12 \kappa}} \sum_{\lambda: \lambda_{1} \leq m} q^{|\lambda|} P_{2 \lambda}\left(1, q, q^{2}, \ldots ; q^{2 n-1}\right) \\
\Phi_{1 b}(m, n ; \tau) & :=q^{\frac{m n(4 m n+2 m+2 n+3)}{12 \kappa}} \sum_{\lambda: \lambda_{1} \leq m} q^{2|\lambda|} P_{2 \lambda}\left(1, q, q^{2}, \ldots ; q^{2 n-1}\right) \\
\Phi_{2}(m, n ; \tau) & :=q^{\frac{m(2 n+1)(2 m n-m+n-1)}{12 \kappa}} \sum_{\lambda: \lambda_{1} \leq m} q^{|\lambda|} P_{2 \lambda}\left(1, q, q^{2}, \ldots ; q^{2 n}\right) \\
\Phi_{3}(m, n ; \tau) & :=q^{\frac{m(2 n-1)(2 m n+n+1)}{12 \kappa}} \sum_{\lambda: \lambda_{1} \leq m} q^{2|\lambda|} P_{2 \lambda}\left(1, q, q^{2}, \ldots ; q^{2 n-2}\right) .
\end{aligned}
$$

Remarks. (1) We note that $\Phi_{3}(m, n ; \tau)$ is not well defined when $n=1$.

(2) We note that the $\kappa_{*}(m, n)$ are odd in the $\mathrm{A}_{2 n}^{(2)}$ cases, and are even for the $\mathrm{C}_{n}^{(1)}$ and $\mathrm{D}_{n+1}^{(2)}$ cases. This dichotomy will be important when seeking pairs of $\Phi_{*}$ whose singular values have ratios that are algebraic integral units.

Our first result concerns the algebraicity of these values and their Galois theoretic properties. We show that these values are in specific abelian extensions of imaginary quadratic fields (see [14, 26] for background on the explicit class field theory of imaginary quadratic fields). For convenience, if $-D<0$ is a discriminant, then we define

$$
D_{0}:= \begin{cases}\frac{D}{4} & \text { if } D \equiv 0 \quad(\bmod 4) \\ \frac{-D-1}{4} & \text { if }-D \equiv 1 \quad(\bmod 4)\end{cases}
$$

Theorem 1.6. Assume the notation above, and let $\kappa:=\kappa_{*}(m, n)$. If $\kappa \tau$ is a CM point with discriminant $-D<0$, then the following are true:

(1) The singular value $\Phi_{*}(m, n ; \tau)$ is an algebraic number.

(2) The multiset

$$
\left\{\Phi_{*}\left(m, n, \tau_{Q} / \kappa\right)_{\left(\gamma \cdot \delta_{Q}(\tau)\right)}^{12 \kappa}:(\gamma, Q) \in W_{\kappa, \tau} \times \mathcal{Q}_{D}\right\}
$$

(see Section 5 for definitions) consists of multiple copies of a Galois orbit over $\mathbb{Q}$. 
(3) If $\kappa>10,|-D|>\kappa^{4} / 2$, and $\operatorname{gcd}\left(D_{0}, \kappa\right)=1$, then the multiset in (2) is a Galois orbit over $\mathbb{Q}$.

Remarks. (1) For each pair of positive integers $m$ and $n$, the inequality in Theorem 1.6 (3) holds for all but finitely many discriminants.

(2) In Section 5 we will show that the values $\Phi_{*}(m, n ; \tau)^{12 \kappa}$ are in a distinguished class field over the ring class field $\mathbb{Q}\left(j\left(\kappa^{2} \tau\right)\right)$, where $j(\tau)$ is the usual Klein $j$-function.

(3) The $\Phi_{*}$ singular values do not in general contain full sets of Galois conjugates. In particular, the singular values in the multiset in Theorem 1.6 (2) generally require $q$-series which are not among the four families $\Phi_{*}$. For instance, only the $i=1$ and $i=m+1$ cases of the Andrews-Gordon identities arise from specializations of $\Phi_{1 a}$ and $\Phi_{1 b}$ respectively. However, the values associated to the other AG identities arise as Galois conjugates of these specializations. One then naturally wonders whether there are even further families of identities, perhaps those which can be uncovered by the theory of complex multiplication. (4) Although Theorem 1.6 (3) indicates that the multiset in (2) is generically a single orbit of Galois conjugates, it turns out that there are indeed situations where the set is more than a single copy of such an orbit. Indeed, the two examples in Section 7 will be such accidents.

We now address the question of singular values and algebraic integral units. Although the singular values of $q^{-1 / 60} G(q)$ and $q^{11 / 60} H(q)$ are not generally algebraic integers, their denominators can be determined exactly, and their ratios always are algebraic integral units. The series $\Phi_{*}$ exhibit similar behavior. The following theorem determines the integrality properties of the singular values. Moreover, it gives algebraic integral unit ratios in the case of the $\mathrm{A}_{2 n}^{(2)}$ identities, generalizing the case of the Rogers-Ramanujan continued fraction.

Theorem 1.7. Assume the notation and hypotheses in Theorem 1.6. Then the following are true:

(1) The singular value $1 / \Phi_{*}(m, n ; \tau)$ is an algebraic integer.

(2) The singular value $\Phi_{*}(m, n ; \tau)$ is a unit over $\mathbb{Z}[1 / \kappa]$.

(3) The ratio $\Phi_{1 a}(m, n ; \tau) / \Phi_{1 b}(m, n ; \tau)$ is an algebraic integral unit.

Remarks. (1) We have that $\Phi_{1 a}(1,1 ; \tau)=q^{-1 / 60} G(q)$ and $\Phi_{1 b}(1,1 ; \tau)=q^{11 / 60} H(q)$. Therefore, Theorem 1.7 (3) implies the theorem of Berndt, Chan, and Zhang that the ratios of these singular values - the singular values of the Rogers-Ramanujan continued fractionare algebraic integral units.

(2) It is natural to ask whether Theorem 1.7(3) is a special property enjoyed only by the $\mathrm{A}_{2 n}^{(2)}$ identities. More precisely, are ratios of singular values of further pairs of $\Phi_{*}$ series algebraic integral units? By Theorem 1.7 (2), it is natural to restrict attention to cases where the $\kappa_{*}(m, n)$ integers agree. Indeed, in these cases the singular values are already integral over the common ring $\mathbb{Z}[1 / \kappa]$. Due to the parity of the $\kappa_{*}(m, n)$, the only other 
cases to consider are pairs involving $\Phi_{2}$ and $\Phi_{3}$. In Section 7 we give an example illustrating that such ratios for $\Phi_{2}$ and $\Phi_{3}$ are not generically algebraic integral units.

Example. In Section 7 we shall consider the $q$-series $\Phi_{1 a}(2,2 ; \tau)$ and $\Phi_{1 b}(2,2 ; \tau)$. For $\tau=$ $\mathrm{i} / 3$, the first 100 coefficients of the $q$-series respectively give the numerical approximations

$$
\begin{aligned}
& \Phi_{1 a}(2,2 ; \mathrm{i} / 3)=0.577350 \cdots \stackrel{?}{=} \frac{1}{\sqrt{3}} \\
& \Phi_{1 b}(2,2 ; \mathrm{i} / 3)=0.125340 \ldots
\end{aligned}
$$

Here we have that $\kappa_{1}(2,2)=9$. Indeed, these values are not algebraic integers. Respectively, they are roots of

$$
\begin{gathered}
3 x^{2}-1 \\
19683 x^{18}-80919 x^{12}+39366 x^{9}+11016 x^{6}+486 x^{3}-1 .
\end{gathered}
$$

However, Theorem 1.7 (2) applies, and we find that $\sqrt{3} \Phi_{1 a}(2,2 ; \mathrm{i} / 3)$ and $\sqrt{3} \Phi_{1 b}(2,2 ; \mathrm{i} / 3)$ are units. Respectively, they are roots of

$$
\begin{gathered}
x-1 \\
x^{18}+6 x^{15}-93 x^{12}-304 x^{9}+420 x^{6}-102 x^{3}+1 .
\end{gathered}
$$

Lastly, Theorem 1.7 (3) applies, and so their ratio

$$
\frac{\Phi_{1 a}(2,2 ; \mathrm{i} / 3)}{\Phi_{1 b}(2,2 ; \mathrm{i} / 3)}=4.60627 \ldots
$$

is a unit. Indeed, it is a root of

$$
x^{18}-102 x^{15}+420 x^{12}-304 x^{9}-93 x^{6}+6 x^{3}+1 .
$$

The remainder of this paper is organized as follows. In Section 2 we recall some basic definitions and facts from the theory of Hall-Littlewood polynomials. We use these facts to give a different combinatorial representation for the left-hand side of (1.12) (see Lemma 2.1). Then, in Sections 3 and 4, we prove Theorems 1.1 1.3 and Theorem 1.5, respectively. The proofs require Weyl denominator formulas, Macdonald identities, and a lemma for $\mathrm{C}_{n}$ hypergeometric series from [8]. We also interpret each of the theorems from the point of view of representation theory. Namely, we explain how these identities correspond to specialized characters of Kac-Moody algebras of affine type.

As noted above, the specializations of the $\theta(a ; q)$ that arise in these identities are essentially modular functions of the type which have been studied extensively by Klein and Siegel. This is the key fact which we employ to derive Theorems 1.6 and 1.7. In Section 5 we recall the Galois theoretic properties of the singular values of Siegel functions as developed by Kubert and Lang, and in Section $[6$ we prove Theorems [1.6] and 1.7. In the last section we conclude with a detailed discussion of examples of Theorems 1.6 and 1.7. 
Note added. One of the referees asked about Rogers-Ramanujan identities for affine Lie algebras other than those considered in this paper. It is indeed possible to extend some of our results to also include $\mathrm{B}_{n}^{(1)}$ and $\mathrm{A}_{2 n-1}^{(2)}$. However, the results of [8] - which are essential in the proofs of Theorems 1.1 1.3 are not strong enough to also deal with these two KacMoody algebras. In [66] Eric Rains and the third author present an alternative method for expressing characters of affine Lie algebras in terms of Hall-Littlewood polynomials to that of [8]. Their method employs what are known as virtual Koornwinder integrals [64, 65] instead of the $\mathrm{C}_{n}$ Bailey lemma used in [8]. This results in several further RogersRamanujan identities, including identities for $\mathrm{B}_{n}^{(1)}$ and $\mathrm{A}_{2 n-1}^{(2)}$. At this stage it is not clear to us how to deal with $\mathrm{D}_{n}^{(1)}$ or any of the exceptional affine Lie algebras.

\section{ACKNOWLEDGEMENTS}

The authors thank Edward Frenkel, James Lepowsky, Dong Hwa Shin, and Drew Sills for their comments on a preliminary version of this paper.

\section{The Hall-Littlewood Polynomials}

Let $\lambda=\left(\lambda_{1}, \lambda_{2}, \ldots\right)$ be an integer partition [3], a nonincreasing sequence of nonnegative integers $\lambda_{1} \geq \lambda_{2} \geq \ldots$ with only finitely nonzero terms. The positive $\lambda_{i}$ are called the parts of $\lambda$, and the number of parts, denoted $l(\lambda)$, is the length of $\lambda$. The size $|\lambda|$ of $\lambda$ is the sum of its parts. The Ferrers-Young diagram of $\lambda$ consists of $l(\lambda)$ left-aligned rows of squares such that the $i$ th row contains $\lambda_{i}$ squares. For example, the Ferrers-Young diagram of $\nu=(6,4,4,2)$ of length 4 and size 16 is

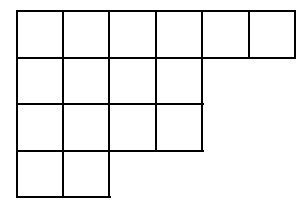

The conjugate partition $\lambda^{\prime}$ corresponds to the transpose of the Ferrers-Young diagram of $\lambda$. For example, we have $\nu^{\prime}=(4,4,3,3,1,1)$. We define nonnegative integers $m_{i}=m_{i}(\lambda)$, for $i \geq 1$, to be the multiplicities of parts of size $i$, so that $|\lambda|=\sum_{i} i m_{i}$. It is easy to see that $m_{i}=\lambda_{i}^{\prime}-\lambda_{i+1}^{\prime}$. We say that a partition is even if its parts are all even. Note that $\lambda^{\prime}$ is even if all multiplicities $m_{i}(\lambda)$ are even. The partition $\nu$ above is an even partition. Given two partitions $\lambda, \mu$ we write $\mu \subseteq \lambda$ if the diagram of $\mu$ is contained in the diagram of $\lambda$, or, equivalently, if $\mu_{i} \leq \lambda_{i}$ for all $i$. To conclude our discussion of partitions, we define the generalized $q$-shifted factorial

$$
b_{\lambda}(q):=\prod_{i \geq 1}(q)_{m_{i}}=\prod_{i \geq 1}(q)_{\lambda_{i}^{\prime}-\lambda_{i+1}^{\prime}} .
$$

Hence, for $\nu$ as above we have $b_{\nu}(q)=(q)_{1}^{2}(q)_{2}$. 
For a fixed positive integer $n$, let $x=\left(x_{1}, \ldots, x_{n}\right)$. Given a partition $\lambda$ such that $l(\lambda) \leq n$, write $x^{\lambda}$ for the monomial $x_{1}^{\lambda_{1}} \ldots x_{n}^{\lambda_{n}}$, and define

$$
v_{\lambda}(q)=\prod_{i=0}^{n} \frac{(q)_{m_{i}}}{(1-q)^{m_{i}}}
$$

where $m_{0}:=n-l(\lambda)$. The Hall-Littlewood polynomial $P_{\lambda}(x ; q)$ is defined as the symmetric function [58]

$$
P_{\lambda}(x ; q)=\frac{1}{v_{\lambda}(q)} \sum_{w \in \mathfrak{S}_{n}} w\left(x^{\lambda} \prod_{i<j} \frac{x_{i}-q x_{j}}{x_{i}-x_{j}}\right),
$$

where the symmetric group $\mathfrak{S}_{n}$ acts on $x$ by permuting the $x_{i}$. It follows from the definition that $P_{\lambda}(x ; q)$ is a homogeneous polynomial of degree $|\lambda|$, a fact used repeatedly in the rest of this paper. $P_{\lambda}(x ; q)$ is defined to be identically 0 if $l(\lambda)>n$. The Hall-Littlewood polynomials may be extended in the usual way to symmetric functions in countably-many variables, see [58].

Here we make this precise when $x$ is specialized to an infinite geometric progression. For $x=\left(x_{1}, x_{2}, \ldots\right)$ not necessarily finite, let $p_{r}$ be the $r$-th power sum symmetric function

$$
p_{r}(x)=x_{1}^{r}+x_{2}^{r}+\cdots,
$$

and $p_{\lambda}=\prod_{i \geq 1} p_{\lambda_{i}}$. The power sums $\left\{p_{\lambda}\left(x_{1}, \ldots, x_{n}\right)\right\}_{l(\lambda) \leq n}$ form a $\mathbb{Q}$-basis of the ring of symmetric functions in $n$ variables. If $\phi_{q}$ denotes the ring homomorphism $\phi_{q}\left(p_{r}\right)=$ $p_{r} /\left(1-q^{r}\right)$, then the modified Hall-Littlewood polynomials $P_{\lambda}^{\prime}(x ; q)$ are defined as the image of the $P_{\lambda}(x ; q)$ under $\phi_{q}$ :

$$
P_{\lambda}^{\prime}=\phi_{q}\left(P_{\lambda}\right)
$$

We also require the Hall-Littlewood polynomials $Q_{\lambda}$ and $Q_{\lambda}^{\prime}$ defined by

$$
Q_{\lambda}(x ; q):=b_{\lambda}(q) P_{\lambda}(x ; q) \quad \text { and } \quad Q_{\lambda}^{\prime}(x ; q):=b_{\lambda}(q) P_{\lambda}^{\prime}(x ; q) \text {. }
$$

Clearly, $Q_{\lambda}^{\prime}=\phi_{q}\left(Q_{\lambda}\right)$.

Up to the point where the $x$-variables are specialized, our proof of Theorems 1.1 1.3 will make use of the modified Hall-Littlewood polynomials, rather than the ordinary HallLittlewood polynomials. Through specialization, we arrive at $P_{\lambda}$ evaluated at a geometric progression thanks to

$$
P_{\lambda}\left(1, q, q^{2}, \ldots ; q^{n}\right)=P_{\lambda}^{\prime}\left(1, q, \ldots, q^{n-1} ; q^{n}\right),
$$

which readily follows from

$$
\phi_{q^{n}}\left(p_{r}\left(1, q, \ldots, q^{n-1}\right)\right)=\frac{1-q^{n r}}{1-q^{r}} \cdot \frac{1}{1-q^{n r}}=p_{r}\left(1, q, q^{2}, \ldots\right) .
$$

From [45, 77] we may infer the following combinatorial formula for the modified HallLittlewood polynomials:

$$
Q_{\lambda}^{\prime}(x ; q)=\sum \prod_{i=1}^{\lambda_{1}} \prod_{a=1}^{n} x_{a}^{\mu_{i}^{(a-1)}-\mu_{i}^{(a)}} q^{\left(\mu_{i}^{(a-1)}-\mu_{i}^{(a)}\right)}\left[\begin{array}{l}
\mu_{i}^{(a-1)}-\mu_{i+1}^{(a)} \\
\mu_{i}^{(a-1)}-\mu_{i}^{(a)}
\end{array}\right]_{q},
$$


where the sum is over partitions $0=\mu^{(n)} \subseteq \cdots \subseteq \mu^{(1)} \subseteq \mu^{(0)}=\lambda^{\prime}$ and

$$
\left[\begin{array}{c}
n \\
m
\end{array}\right]_{q}= \begin{cases}\frac{(q)_{n}}{(q)_{m}(q)_{n-m}} & \text { if } m \in\{0,1, \ldots, n\} \\
0 & \text { otherwise }\end{cases}
$$

is the usual $q$-binomial coefficient. Therefore, by (2.1)-(2.5), we have obtained the following combinatorial description of the $q$-series we have assembled from the Hall-Littlewood polynomials.

Lemma 2.1. If $m$ and $n$ are positive integers, then

$$
\begin{aligned}
\sum_{\substack{\lambda \\
\lambda_{1} \leq m}} q^{(\sigma+1)|\lambda|} & P_{2 \lambda}\left(1, q, q^{2}, \ldots ; q^{n}\right) \\
& =\sum \prod_{i=1}^{2 m}\left\{\frac{q^{\frac{1}{2}(\sigma+1) \mu_{i}^{(0)}}}{\left(q^{n} ; q^{n}\right)_{\mu_{i}^{(0)}-\mu_{i+1}^{(0)}}} \prod_{a=1}^{n} q^{\mu_{i}^{(a)}+n\left(\left(_{i}^{(a-1)}{ }_{2}^{(a)}\right)\right.}\left[\begin{array}{c}
\mu_{i}^{(a-1)}-\mu_{i+1}^{(a)} \\
\mu_{i}^{(a-1)}-\mu_{i}^{(a)}
\end{array}\right]_{q^{n}}\right\},
\end{aligned}
$$

where the sum on the right is over partitions $0=\mu^{(n)} \subseteq \cdots \subseteq \mu^{(1)} \subseteq \mu^{(0)}$ such that $\left(\mu^{(0)}\right)^{\prime}$ is even and $l\left(\mu^{(0)}\right) \leq 2 m$.

Lemma 2.1 may be used to express the sum sides of (1.7)-(1.12) combinatorially. Moreover, we have that (2.6) generalizes the sums in (1.1), (1.2), and (1.5). To see this, we note that the above simplifies for $n=1$ to

$$
\sum_{\substack{\lambda \\ \lambda_{1} \leq m}} q^{(\sigma+1)|\lambda|} P_{2 \lambda}\left(1, q, q^{2}, \ldots ; q\right)=\sum \prod_{i=1}^{2 m} \frac{q^{\frac{1}{2} \mu_{i}\left(\mu_{i}+\sigma\right)}}{(q)_{\mu_{i}-\mu_{i+1}}}
$$

summed on the right over partitions $\mu$ of length at most $2 m$ whose conjugates are even. Such partitions are characterized by the restriction $\mu_{2 i}=\mu_{2 i-1}=: r_{i}$ so that we get

$$
\sum_{\substack{\lambda \\ \lambda_{1} \leq m}} q^{(\sigma+1)|\lambda|} P_{2 \lambda}\left(1, q, q^{2}, \ldots ; q\right)=\sum_{r_{1} \geq \cdots \geq r_{m} \geq 0} \prod_{i=1}^{m} \frac{q^{r_{i}\left(r_{i}+\sigma\right)}}{(q)_{r_{i}-r_{i+1}}}
$$

in accordance with (1.5).

If instead we consider $m=1$ and replace $\mu^{(j)}$ by $\left(r_{j}, s_{j}\right)$ for $j \geq 0$, we find

$$
\begin{aligned}
& \sum_{r=0}^{\infty} q^{(\sigma+1) r} P_{\left(2^{r}\right)}\left(1, q, q^{2}, \ldots ; q^{n}\right) \\
& \quad=\sum \frac{q^{(\sigma+1) r_{0}}}{\left(q^{n} ; q^{n}\right)_{r_{0}}} \prod_{j=1}^{n} q^{r_{j}+s_{j}+n\left({ }_{2}^{r_{j-1}-r_{j}}\right)+n\left({ }_{2}^{s_{j-1}-s_{j}}\right)}\left[\begin{array}{c}
r_{j-1}-s_{j} \\
r_{j-1}-r_{j}
\end{array}\right]_{q^{n}}\left[\begin{array}{c}
s_{j-1} \\
s_{j}
\end{array}\right]_{q^{n}} \\
& \quad=\frac{\left(q^{n+4} ; q^{n+4}\right)_{\infty}}{(q)_{\infty}} \cdot \theta\left(q^{2-\sigma} ; q^{n+4}\right),
\end{aligned}
$$

where the second sum is over $r_{0}, s_{0}, \ldots, r_{n-1}, s_{n-1}$ such that $r_{0}=s_{0}$, and $r_{n}=s_{n}:=0$. 
We conclude this section with a remark about Theorem 1.5. Due to the occurrence of the limit, the left-hand side does not take the form of the usual sum-side of a RogersRamanujan-type identity. For special cases it is, however, possible to eliminate the limit. For example, for partitions of the form $\left(2^{r}\right)$ we found that

$$
P_{\left(2^{r}\right)}\left(1, q, q^{2}, \ldots ; q^{2 n+\delta}\right)=\sum_{r \geq r_{1} \geq \cdots \geq r_{n} \geq 0} \frac{q^{r^{2}-r+r_{1}^{2}+\cdots+r_{n}^{2}+r_{1}+\cdots+r_{n}}}{(q)_{r-r_{1}}(q)_{r_{1}-r_{2}} \cdots(q)_{r_{n-1}-r_{n}}\left(q^{2-\delta} ; q^{2-\delta}\right)_{r_{n}}}
$$

for $\delta=0,1$. This turns the $m=2$ case of Theorem [1.5 into

$$
\sum_{r_{1} \geq \cdots \geq r_{n} \geq 0} \frac{q^{r_{1}^{2}+\cdots+r_{n}^{2}+r_{1}+\cdots+r_{n}}}{(q)_{r_{1}-r_{2}} \cdots(q)_{r_{n-1}-r_{n}}\left(q^{2-\delta} ; q^{2-\delta}\right)_{r_{n}}}=\frac{\left(q^{2 n+2+\delta} ; q^{2 n+2+\delta}\right)_{\infty}}{(q)_{\infty}} \cdot \theta\left(q ; q^{2 n+2+\delta}\right) .
$$

For $\delta=1$ this is the $i=1$ case of the Andrews-Gordon identity (1.5) (with $m$ replaced by $n$ ). For $\delta=0$ it corresponds to the $i=1$ case of (1.11). We do not know how to generalize (2.7) to arbitrary rectangular shapes.

\section{Proof of Theorems 1.11 .3}

Here we prove Theorems 1.1 1.3. We begin by recalling key aspects of the classical works of Andrews and Watson which give hints of the generalizations we obtain.

3.1. The Watson-Andrews approach. In 1929 Watson proved the Rogers-Ramanujan identities (1.1) and (1.2) by first proving a new basic hypergeometric series transformation between a terminating balanced ${ }_{4} \phi_{3}$ series and a terminating very-well-poised ${ }_{8} \phi_{7}$ series 75 .

$$
\begin{aligned}
& \frac{(a q, a q / b c)_{N}}{(a q / b, a q / c)_{N}} \sum_{r=0}^{N} \frac{\left(b, c, a q / d e, q^{-N}\right)_{r}}{\left(q, a q / d, a q / e, b c q^{-N} / a\right)_{r}} q^{r} \\
& =\sum_{r=0}^{N} \frac{1-a q^{2 r}}{1-a} \cdot \frac{\left(a, b, c, d, e, q^{-N}\right)_{r}}{(q, a q / b, a q / c, a q / d, a q / e)_{r}} \cdot\left(\frac{a^{2} q^{N+2}}{b c d e}\right)^{r} .
\end{aligned}
$$

Here $a, b, c, d, e$ are indeterminates, $N$ is a nonnegative integer and

$$
\left(a_{1}, \ldots, a_{m}\right)_{k}:=\left(a_{1}, \ldots, q_{m} ; q\right)=\left(a_{1} ; q\right)_{k} \cdots\left(a_{m} ; q\right)_{k} .
$$

By letting $b, c, d$, e tend to infinity and taking the nonterminating limit $N \rightarrow \infty$, Watson arrived at what is known as the Rogers-Selberg identity [70, 72]

$$
\sum_{r=0}^{\infty} \frac{a^{r} q^{r^{2}}}{(q)_{r}}=\frac{1}{(a q)_{\infty}} \sum_{r=0}^{\infty} \frac{1-a q^{2 r}}{1-a} \cdot \frac{(a)_{r}}{(q)_{r}} \cdot(-1)^{r} a^{2 r} q^{5\left(\begin{array}{c}
r \\
2
\end{array}\right)+2 r}
$$

\footnotetext{
${ }^{3}$ Here and elsewhere in the paper we ignore questions of convergence. From an analytic point of view, the transition from (3.1) to (3.2) requires the use of the dominated convergence theorem, imposing the restriction $|q|<1$ on the Rogers-Selberg identity. We however choose to view this identity as an identity between formal power series in $q$, in line with the combinatorial and representation-theoretic interpretations of Rogers-Ramanujan-type identities.
} 
For $a=1$ or $a=q$ the sum on the right can be expressed in product-form by the Jacobi triple-product identity

$$
\sum_{r=-\infty}^{\infty}(-1)^{r} x^{r} q^{\left(\begin{array}{c}
r \\
2
\end{array}\right)}=(q)_{\infty} \cdot \theta(x ; q)
$$

resulting in (1.1) and (1.2).

Almost 50 years after Watson's work, Andrews showed that the Andrews-Gordon identities (1.5) for $i=1$ and $i=m+1$ follow in a similar way from a multiple series generalization of (3.1) in which the ${ }_{8} \phi_{7}$ series on the right is replaced by a terminating very-well-poised ${ }_{2 m+6} \phi_{2 m+5}$ series depending on $2 m+2$ parameters instead of $b, c, d, e$ [2]. Again the key steps are to let all these parameters tend to infinity, to take the nonterminating limit, and to then express the $a=1$ or $a=q$ instances of the resulting sum as a product by the Jacobi triple-product identity.

Recently, Bartlett and the third author obtained an analog of Andrews' multiple series transformation for the $\mathrm{C}_{n}$ root system [8, Theorem 4.2]. Apart from the variables $\left(x_{1}, \ldots, x_{n}\right)$ - which play the role of $a$ in (3.1), and are related to the underlying root system - the $\mathrm{C}_{n}$ Andrews transformation again contains $2 m+2$ parameters. Unfortunately, simply following the Andrews-Watson procedure is no longer sufficient. In 61] Milne already obtained the $\mathrm{C}_{n}$ analogue of the Rogers-Selberg identity (3.2) (the $m=1$ case of (3.3) below) and considered specializations along the lines of Andrews and Watson. Only for $\mathrm{C}_{2}$ did this result in a Rogers-Ramanujan-type identity: the modulus 6 case of (1.10) mentioned previously.

The first two steps towards a proof of (1.7)-(1.12), however, are the same as those of Watson and Andrews: we let all $2 m+2$ parameters in the $\mathrm{C}_{n}$ Andrews transformation tend to infinity and take the nonterminating limit. Then, as shown in [8], the right-hand side can be expressed in terms of modified Hall-Littlewood polynomials, resulting in the level- $m \mathrm{C}_{n}$ Rogers-Selberg identity

$$
\sum_{\substack{\lambda \\ \lambda_{1} \leq m}} q^{|\lambda|} P_{2 \lambda}^{\prime}(x ; q)=L_{m}^{(0)}(x ; q),
$$

where

$$
L_{m}^{(0)}(x ; q):=\sum_{r \in \mathbb{Z}_{+}^{n}} \frac{\Delta_{\mathrm{C}}\left(x q^{r}\right)}{\Delta_{\mathrm{C}}(x)} \prod_{i=1}^{n} x_{i}^{2(m+1) r_{i}} q^{(m+1) r_{i}^{2}+n\left(\begin{array}{c}
r_{i} \\
2
\end{array}\right)} \cdot \prod_{i, j=1}^{n}\left(-\frac{x_{i}}{x_{j}}\right)^{r_{i}} \frac{\left(x_{i} x_{j}\right)_{r_{i}}}{\left(q x_{i} / x_{j}\right)_{r_{i}}} .
$$

Here we have that

$$
\Delta_{\mathrm{C}}(x):=\prod_{i=1}^{n}\left(1-x_{i}^{2}\right) \prod_{1 \leq i<j \leq n}\left(x_{i}-x_{j}\right)\left(x_{i} x_{j}-1\right)
$$

is the $\mathrm{C}_{n}$ Vandermonde product, and $f\left(x q^{r}\right)$ is shorthand for $f\left(x_{1} q^{r_{1}}, \ldots, x_{n} q^{r_{n}}\right)$.

Remark. As mentioned previously, (3.3) for $m=1$ is Milne's $\mathrm{C}_{n}$ Rogers-Selberg formula [61, Corollary 2.21]. 
The strategy for the proofs of Theorems 1.1 1.3 is now simple to describe. By comparing the left-hand side of (3.3) with that of (1.7) $-(1.10)$, it follows that we should make the simultaneous substitutions

$$
q \mapsto q^{n}, \quad x_{i} \mapsto q^{(n+\sigma+1) / 2-i} \quad(1 \leq i \leq n) .
$$

Then, by the homogeneity and symmetry of the (modified) Hall-Littlewood polynomials and (2.5), we have

$$
\sum_{\substack{\lambda \\ \lambda_{1} \leq m}} q^{|\lambda|} P_{2 \lambda}^{\prime}(x ; q) \longmapsto \sum_{\substack{\lambda \\ \lambda_{1} \leq m}} q^{(\sigma+1)|\lambda|} P_{2 \lambda}\left(1, q, q^{2}, \ldots ; q^{n}\right) .
$$

Therefore, we wish to carry out these maneuvers and prove that the resulting right-hand side can be described as a product of modified theta functions in the four families in the theorems. The problem we face is that making the substitutions (3.4) in the right-hand side of (3.3) and then writing the resulting $q$-series in product form is very difficult.

To get around this problem, we take a rather different route and (up to a small constant) first double the rank of the underlying $\mathrm{C}_{n}$ root system and then take a limit in which products of pairs of $x$-variables tend to one. To do so we require another result from [8].

First we extend our earlier definition of the $q$-shifted factorial to

$$
(a)_{k}=(a)_{\infty} /\left(a q^{k}\right)_{\infty}
$$

Importantly, we note that $1 /(q)_{k}=0$ for $k$ a negative integer. Then, for $x=\left(x_{1}, \ldots, x_{n}\right)$, $p$ an integer such that $0 \leq p \leq n$ and $r \in \mathbb{Z}^{n}$, we have

$$
\begin{aligned}
L_{m}^{(p)}(x ; q):=\sum_{r \in \mathbb{Z}^{n}} \frac{\Delta_{\mathrm{C}}\left(x q^{r}\right)}{\Delta_{\mathrm{C}}(x)} \prod_{i=1}^{n} x_{i}^{2(m+p+1) r_{i}} q^{(m+1) r_{i}^{2}+(n+p)\left(\begin{array}{c}
r_{i} \\
2
\end{array}\right)} & \\
& \times \prod_{i=1}^{n} \prod_{j=p+1}^{n}\left(-\frac{x_{i}}{x_{j}}\right)^{r_{i}} \frac{\left(x_{i} x_{j}\right)_{r_{i}}}{\left(q x_{i} / x_{j}\right)_{r_{i}}} .
\end{aligned}
$$

Note that the summand of $L_{m}^{(p)}(x ; q)$ vanishes if one of $r_{p+1}, \ldots, r_{n}<0$.

The following lemma will be crucial for our strategy to work.

Lemma 3.1 ([8, Lemma A.1]). For $1 \leq p \leq n-1$,

$$
\lim _{x_{p+1} \rightarrow x_{p}^{-1}} L_{m}^{(p-1)}(x ; q)=L_{m}^{(p)}\left(x_{1}, \ldots, x_{p-1}, x_{p+1}, \ldots, x_{n} ; q\right) .
$$

This will be the key to the proof of the generalized Rogers-Ramanujan identities of Theorems 1.1 1.3 although the level of difficulty varies considerably from case to case.

We begin with the simplest proof, that of the $\mathrm{C}_{n}$ Rogers-Ramanujan and AndrewsGordon identities of Theorem 1.2. Although this theorem may also be proved more directly by principally specializing [8, Theorem 1.1] (more on this later), we take a more indirect approach in order to describe the general method using the simplest available example. For the $\mathrm{A}_{2 n}^{(2)}$ and $\mathrm{D}_{n+1}^{(2)}$ Rogers-Ramanujan and Andrews-Gordon identities we have no 
analogues of [8, Theorem 1.1], and in these cases we rely on to the method described below.

3.2. Proof of Theorem 1.2. Here we carry out the strategy described in the previous section by making use of the $\mathrm{C}_{n}$ and $\mathrm{B}_{n}$ Weyl denominator formulas, and the $\mathrm{D}_{n+1}^{(2)}$ Macdonald identity.

Proof of Theorem 1.2. By iterating (3.7), we have

$$
\lim _{y_{1} \rightarrow x_{1}^{-1}} \ldots \lim _{y_{n} \rightarrow x_{n}^{-1}} L_{m}^{(0)}\left(x_{1}, y_{1}, \ldots, x_{n}, y_{n}\right)=L_{m}^{(n)}\left(x_{1}, \ldots, x_{n}\right) .
$$

Hence, after replacing $x \mapsto\left(x_{1}, y_{1}, \ldots, x_{n}, y_{n}\right)$ in (3.3) (which corresponds to the doubling of the rank mentioned previously) and taking the $y_{i} \rightarrow x_{i}^{-1}$ limit for $1 \leq i \leq n$, we find

$$
\begin{aligned}
\sum_{\substack{\lambda \\
\lambda_{1} \leq m}} q^{|\lambda|} P_{2 \lambda}^{\prime}\left(x^{ \pm} ; q\right)=\frac{1}{(q)_{\infty}^{n} \prod_{i=1}^{n} \theta\left(x_{i}^{2} ; q\right) \prod_{1 \leq i<j \leq n} \theta\left(x_{i} / x_{j}, x_{i} x_{j} ; q\right)} \\
\quad \times \sum_{r \in \mathbb{Z}^{n}} \Delta_{\mathrm{C}}\left(x q^{r}\right) \prod_{i=1}^{n} x_{i}^{\kappa r_{i}-i+1} q^{\frac{1}{2} \kappa r_{i}^{2}-n r_{i}},
\end{aligned}
$$

where $\kappa=2 m+2 n+2$ and $f\left(x^{ \pm}\right)=f\left(x_{1}, x_{1}^{-1}, \ldots, x_{n}, x_{n}^{-1}\right)$. Next we make the simultaneous substitutions

$$
q \mapsto q^{2 n}, \quad x_{i} \mapsto q^{n-i+1 / 2}=: \hat{x}_{i}(1 \leq i \leq n),
$$

which corresponds to (3.4) with $(n, \sigma) \mapsto(2 n, 0)$. By the identity

$$
\left(q^{2 n} ; q^{2 n}\right)_{\infty}^{n} \cdot \prod_{i=1}^{n} \theta\left(q^{2 n-2 i+1} ; q^{2 n}\right) \cdot \prod_{1 \leq i<j \leq n} \theta\left(q^{j-i}, q^{2 n-i-j+1} ; q^{2 n}\right)=\frac{(q)_{\infty}^{n+1}}{\left(q^{2} ; q^{2}\right)_{\infty}}
$$

and

$$
\begin{aligned}
q^{2 n|\lambda|} & P_{2 \lambda}^{\prime}\left(q^{n-1 / 2}, q^{1 / 2-n}, \ldots, q^{1 / 2}, q^{-1 / 2} ; q^{2 n}\right) & & \\
& =q^{2 n|\lambda|} P_{2 \lambda}^{\prime}\left(q^{1 / 2-n}, q^{3 / 2-n}, \ldots, q^{n-1 / 2} ; q^{2 n}\right) & & \text { by symmetry } \\
& =q^{|\lambda|} P_{2 \lambda}^{\prime}\left(1, q, \ldots, q^{2 n-1} ; q^{2 n}\right) & & \text { by homogeneity } \\
& =q^{|\lambda|} P_{2 \lambda}\left(1, q, q^{2}, \ldots ; q^{2 n}\right) & & \text { by (2.5), }
\end{aligned}
$$

we obtain

$$
\sum_{\substack{\lambda \\ \lambda_{1} \leq m}} q^{|\lambda|} P_{2 \lambda}\left(1, q, q^{2}, \ldots ; q^{2 n}\right)=\frac{\left(q^{2} ; q^{2}\right)_{\infty}}{(q)_{\infty}^{n+1}} \mathscr{M}
$$

where

$$
\mathscr{M}:=\sum_{r \in \mathbb{Z}^{n}} \Delta_{\mathrm{C}}\left(\hat{x} q^{2 n r}\right) \prod_{i=1}^{n} \hat{x}_{i}^{\kappa r_{i}-i+1} q^{n \kappa r_{i}^{2}-2 n^{2} r_{i}}
$$


We must express $\mathscr{M}$ in product form. As a first step, we use the $\mathrm{C}_{n}$ Weyl denominator formula [46, Lemma 2]

$$
\Delta_{\mathrm{C}}(x)=\operatorname{det}_{1 \leq i, j \leq n}\left(x_{i}^{j-1}-x_{i}^{2 n-j+1}\right),
$$

as well as multilinearity, to write $\mathscr{M}$ as

$$
\mathscr{M}=\operatorname{det}_{1 \leq i, j \leq n}\left(\sum_{r \in \mathbb{Z}} \hat{x}_{i}^{\kappa r-i+1} q^{n \kappa r^{2}-2 n^{2} r}\left(\left(\hat{x}_{i} q^{2 n r}\right)^{j-1}-\left(\hat{x}_{i} q^{2 n r}\right)^{2 n-j+1}\right)\right) .
$$

We now replace $(i, j) \mapsto(n-j+1, n-i+1)$ and, viewing the resulting determinant as being of the form $\operatorname{det}\left(\sum_{r} u_{i j ; r}-\sum_{r} v_{i j ; r}\right)$, we change the summation index $r \mapsto-r-1$ in the sum over $v_{i j ; r}$. Then we find that

$$
\mathscr{M}=\operatorname{det}_{1 \leq i, j \leq n}\left(q^{a_{i j}} \sum_{r \in \mathbb{Z}} y_{i}^{2 n r-i+1} q^{2 n \kappa\left(\begin{array}{l}
r \\
2
\end{array}\right)+\frac{1}{2} \kappa r}\left(\left(y_{i} q^{\kappa r}\right)^{j-1}-\left(y_{i} q^{\kappa r}\right)^{2 n-j}\right)\right),
$$

where $y_{i}=q^{\kappa / 2-i}$ and $a_{i j}=j^{2}-i^{2}+(i-j)(\kappa+1) / 2$. Since the factor $q^{a_{i j}}$ does not contribute to the determinant, we can apply the $\mathrm{B}_{n}$ Weyl denominator formula [46]

$$
\operatorname{det}_{1 \leq i, j \leq n}\left(x_{i}^{j-1}-x_{i}^{2 n-j}\right)=\prod_{i=1}^{n}\left(1-x_{i}\right) \prod_{1 \leq i<j \leq n}\left(x_{i}-x_{j}\right)\left(x_{i} x_{j}-1\right)=: \Delta_{\mathrm{B}}(x)
$$

to obtain

$$
\mathscr{M}=\sum_{r \in \mathbb{Z}^{n}} \Delta_{\mathrm{B}}\left(y q^{\kappa r}\right) \prod_{i=1}^{n} y_{i}^{2 n r_{i}-i+1} q^{2 n \kappa\left(\begin{array}{c}
r_{i} \\
2
\end{array}\right)+\frac{1}{2} \kappa r_{i}}
$$

By the $\mathrm{D}_{n+1}^{(2)}$ Macdonald identity [57]

$$
\begin{aligned}
\sum_{r \in \mathbb{Z}^{n}} \Delta_{\mathrm{B}}\left(x q^{r}\right) \prod_{i=1}^{n} x_{i}^{2 n r_{i}-i+1} q^{2 n}\left(\begin{array}{c}
r_{i} \\
2
\end{array}\right)+\frac{1}{2} r_{i} \\
=\left(q^{1 / 2} ; q^{1 / 2}\right)_{\infty}(q)_{\infty}^{n-1} \prod_{i=1}^{n} \theta\left(x_{i} ; q^{1 / 2}\right)_{\infty} \prod_{1 \leq i<j \leq n} \theta\left(x_{i} / x_{j}, x_{i} x_{j} ; q\right)
\end{aligned}
$$

with $(q, x) \mapsto\left(q^{\kappa}, y\right)$ this yields

$$
\mathscr{M}=\left(q^{\kappa / 2} ; q^{\kappa / 2}\right)_{\infty}\left(q^{\kappa} ; q^{\kappa}\right)_{\infty}^{n-1} \prod_{i=1}^{n} \theta\left(q^{i} ; q^{\kappa / 2}\right) \prod_{1 \leq i<j \leq n} \theta\left(q^{j-i}, q^{i+j} ; q^{\kappa}\right),
$$

where we have also used the simple symmetry $\theta\left(q^{a-b} ; q^{a}\right)=\theta\left(q^{b} ; q^{a}\right)$. Substituting (3.15) into (3.10) proves the first equality of (1.9).

Establishing the second equality is a straightforward exercise in manipulating infinite products, and we omit the details. 
There is a somewhat different approach to (1.9) based on the representation theory of the affine Kac-Moody algebra $\mathrm{C}_{n}^{(1)}$ [43]. Let $I=\{0,1, \ldots, n\}$, and $\alpha_{i}, \alpha_{i}^{\vee}$ and $\Lambda_{i}$ for $i \in I$ the simple roots, simple coroots and fundamental weights of $\mathrm{C}_{n}^{(1)}$. Let $\langle\cdot, \cdot\rangle$ denote the usual pairing between the Cartan subalgebra $\mathfrak{h}$ and its dual $\mathfrak{h}^{*}$, so that $\left\langle\Lambda_{i}, \alpha_{j}^{\vee}\right\rangle=\delta_{i j}$. Finally, let $V(\Lambda)$ be the integrable highest-weight module of $\mathrm{C}_{n}^{(1)}$ of highest weight $\Lambda$ with character $\operatorname{ch} V(\Lambda)$.

The homomorphism

$$
F_{\mathbb{1}}: \mathbb{C}\left[\left[\mathrm{e}^{-\alpha_{0}}, \ldots, \mathrm{e}^{-\alpha_{n}}\right]\right] \rightarrow \mathbb{C}[[q]], \quad F_{1}\left(\mathrm{e}^{-\alpha_{i}}\right)=q \text { for all } i \in I
$$

is known as principal specialization [48]. Subject to this specialization, $\operatorname{ch} V(\Lambda)$ admits a simple product form as follows. Let $\rho$ be the Weyl vector (that is $\left\langle\rho, \alpha_{i}^{\vee}\right\rangle=1$ for $i \in I$ ) and $\operatorname{mult}(\alpha)$ the multiplicity of $\alpha$. Then [42, 49, we have

$$
F_{\mathbb{1}}\left(\mathrm{e}^{-\Lambda} \operatorname{ch} V(\Lambda)\right)=\prod_{\alpha \in \Delta_{+}^{\vee}}\left(\frac{1-q^{\langle\Lambda+\rho, \alpha\rangle}}{1-q^{\langle\rho, \alpha\rangle}}\right)^{\operatorname{mult}(\alpha)},
$$

where $\Delta_{+}^{\vee}$ is the set of positive coroots. This result, which is valid for all types $\mathrm{X}_{N}^{(r)}$, can be rewritten in terms of theta functions. Assuming $\mathrm{C}_{n}^{(1)}$ and setting

$$
\Lambda=\left(\lambda_{0}-\lambda_{1}\right) \Lambda_{0}+\left(\lambda_{1}-\lambda_{2}\right) \Lambda_{1}+\cdots+\left(\lambda_{n-1}-\lambda_{n}\right) \Lambda_{n-1}+\lambda_{n} \Lambda_{n}
$$

for $\lambda=\left(\lambda_{0}, \lambda_{1}, \ldots, \lambda_{n}\right)$ a partition, this rewriting takes the form

$$
\begin{aligned}
F_{\mathbb{1}}\left(\mathrm{e}^{-\Lambda} \operatorname{ch} V(\Lambda)\right) & =\frac{\left(q^{2} ; q^{2}\right)_{\infty}\left(q^{\kappa / 2} ; q^{\kappa / 2}\right)_{\infty}\left(q^{\kappa} ; q^{\kappa}\right)_{\infty}^{n-1}}{(q ; q)_{\infty}^{n+1}} \\
& \times \prod_{i=1}^{n} \theta\left(q^{\lambda_{i}+n-i+1} ; q^{\kappa / 2}\right) \prod_{1 \leq i<j \leq n} \theta\left(q^{\lambda_{i}-\lambda_{j}-i+j}, q^{\lambda_{i}+\lambda_{j}+2 n+2-i-j} ; q^{\kappa}\right),
\end{aligned}
$$

where $\kappa=2 n+2 \lambda_{0}+2$.

The earlier product form now arises by recognizing (see e.g., [8, Lemma 2.1]) the righthand side of (3.8) as

$$
\mathrm{e}^{-m \Lambda_{0}} \operatorname{ch} V\left(m \Lambda_{0}\right)
$$

upon the identification

$$
q=\mathrm{e}^{-\alpha_{0}-2 \alpha_{1}-\cdots-2 \alpha_{n-1}-\alpha_{n}} \quad \text { and } \quad x_{i}=\mathrm{e}^{-\alpha_{i}-\cdots-\alpha_{n-1}-\alpha_{n} / 2} \quad(1 \leq i \leq n) .
$$

Indeed, the equality between the left-hand side of (3.8) and (3.20) is exactly the first part of the previously mentioned [8, Theorem 1.1]. Since (3.9) corresponds exactly to the 
principal specialization (3.16), it follows from (3.19) with $\lambda=\left(m, 0^{n}\right)$, that

$$
\begin{gathered}
F_{\mathbb{1}}\left(\mathrm{e}^{-m \Lambda_{0}} \operatorname{ch} V\left(m \Lambda_{0}\right)\right)=\frac{\left(q^{2} ; q^{2}\right)_{\infty}\left(q^{\kappa / 2} ; q^{\kappa / 2}\right)_{\infty}\left(q^{\kappa} ; q^{\kappa}\right)_{\infty}^{n-1}}{(q ; q)_{\infty}^{n+1}} \\
\times \prod_{i=1}^{n+1} \theta\left(q^{n-i+1} ; q^{\kappa / 2}\right) \prod_{1 \leq i<j \leq n} \theta\left(q^{j-i}, q^{i+j} ; q^{\kappa}\right) .
\end{gathered}
$$

This representation-theoretic approach is not essentially different from our earlier $q$-series proof. The principal specialization formula (3.19) itself is an immediate consequence of the $\mathrm{D}_{n+1}^{(2)}$ Macdonald identity, and if instead of the right-hand side of (3.8) we consider the more general

$$
\begin{aligned}
\mathrm{e}^{-\Lambda} \operatorname{ch} V(\Lambda)= & \frac{1}{(q)_{\infty}^{n} \prod_{i=1}^{n} \theta\left(x_{i}^{2} ; q\right) \prod_{1 \leq i<j \leq n} \theta\left(x_{i} / x_{j}, x_{i} x_{j} ; q\right)} \\
& \times \sum_{r \in \mathbb{Z}^{n}} \operatorname{det}_{1 \leq i, j \leq n}\left(\left(x_{i} q^{r_{i}}\right)^{j-\lambda_{j}-1}-\left(x_{i} q^{r_{i}}\right)^{2 n-j+\lambda_{j}+1}\right) \prod_{i=1}^{n} x_{i}^{\kappa r_{i}+\lambda_{i}-i+1} q^{\frac{1}{2} \kappa r_{i}^{2}-n r_{i}}
\end{aligned}
$$

for $\kappa=2 n+2 \lambda_{0}+2$, then all of the steps carried out between (3.8) and (3.15) carry over to this more general setting. The only notable changes are that (3.12) generalizes to

$$
\mathscr{M}=\operatorname{det}_{1 \leq i, j \leq n}\left(\sum_{r \in \mathbb{Z}} \hat{x}_{i}^{\kappa r+\lambda_{i}-i+1} q^{n \kappa r^{2}-2 n^{2} r} \cdot\left(\left(\hat{x}_{i} q^{2 n r}\right)^{j-\lambda_{j}-1}-\left(\hat{x}_{i} q^{2 n r}\right)^{2 n-j+\lambda_{j}+1}\right)\right),
$$

and that in (3.13) we have to redefine $y_{i}$ as $q^{\kappa / 2-\lambda_{n-i+1}-i}$, and $a_{i j}$ as

$$
j^{2}-i^{2}+(i-j)(\kappa+1) / 2+(j-1 / 2) \lambda_{n-j+1}-(i-1 / 2) \lambda_{n-i+1} .
$$

3.3. Proof of Theorem 1.1 (1.7a). Here we prove (1.7a) by making use of the $\mathrm{B}_{n}^{(1)}$ Macdonald identity.

Proof of Theorem 1.1(1.7a). Again we iterate (3.7), but this time the variable $x_{n}$, remains unpaired:

$$
\lim _{y_{1} \rightarrow x_{1}^{-1}} \ldots \lim _{y_{n-1} \rightarrow x_{n-1}^{-1}} L_{m}^{(0)}\left(x_{1}, y_{1}, \ldots, x_{n-1}, y_{n-1}, x_{n}\right)=L_{m}^{(n-1)}\left(x_{1}, \ldots, x_{n}\right) .
$$

Therefore, if we replace $x \mapsto\left(x_{1}, y_{1}, \ldots, x_{n-1}, y_{n-1}, x_{n}\right)$ in (3.3) (changing the rank from $n$ to $2 n-1)$ and take the $y_{i} \rightarrow x_{i}^{-1}$ limit for $1 \leq i \leq n-1$, we obtain

$$
\begin{aligned}
\sum_{\substack{\lambda \\
\lambda_{1} \leq m}} q^{|\lambda|} & P_{2 \lambda}^{\prime}\left(x_{1}^{ \pm}, \ldots, x_{n-1}^{ \pm}, x_{n} ; q\right) \\
= & \frac{1}{(q)_{\infty}^{n-1}\left(q x_{n}^{2}\right)_{\infty} \prod_{i=1}^{n-1}\left(q x_{i}^{ \pm} x_{n}, q x_{i}^{ \pm 2}\right)_{\infty} \prod_{1 \leq i<j \leq n-1}\left(q x_{i}^{ \pm} x_{j}^{ \pm}\right)_{\infty}} \\
& \quad \times \sum_{r \in \mathbb{Z}^{n}} \frac{\Delta_{\mathrm{C}}\left(x q^{r}\right)}{\Delta_{\mathrm{C}}(x)} \prod_{i=1}^{n}\left(-\frac{x_{i}^{\kappa}}{x_{n}}\right)^{r_{i}} q^{\frac{1}{2} \kappa r_{i}^{2}-\frac{1}{2}(2 n-1) r_{i}} \frac{\left(x_{i} x_{n}\right)_{r_{i}}}{\left(q x_{i} / x_{n}\right)_{r_{i}}}
\end{aligned}
$$


where $\kappa=2 m+2 n+1,\left(a x_{i}^{ \pm}\right)_{\infty}:=\left(a x_{i}\right)_{\infty}\left(a x_{i}^{-1}\right)_{\infty}$ and

$$
\left(a x_{i}^{ \pm} x_{j}^{ \pm}\right)_{\infty}:=\left(a x_{i} x_{j}\right)_{\infty}\left(a x_{i}^{-1} x_{j}\right)_{\infty}\left(a x_{i} x_{j}^{-1}\right)_{\infty}\left(a x_{i}^{-1} x_{j}^{-1}\right)_{\infty} .
$$

Recalling the comment immediately after (3.6), the summand of (3.21) vanishes unless $r_{n} \geq 0$.

Let $\hat{x}:=\left(-x_{1}, \ldots,-x_{n-1},-1\right)$ and

$$
\phi_{r}= \begin{cases}1 & \text { if } r=0 \\ 2 & \text { if } r=1,2, \ldots\end{cases}
$$

Letting $x_{n}$ tend to 1 in (3.21), and using

$$
\lim _{x_{n} \rightarrow 1} \frac{\Delta_{\mathrm{C}}\left(x q^{r}\right)}{\Delta_{\mathrm{C}}(x)} \prod_{i=1}^{n} \frac{\left(x_{i} x_{n}\right)_{r_{i}}}{\left(q x_{i} / x_{n}\right)_{r_{i}}}=\phi_{r_{n}} \frac{\Delta_{\mathrm{B}}\left(\hat{x} q^{r}\right)}{\Delta_{\mathrm{B}}(\hat{x})},
$$

we find that

$$
\begin{aligned}
\sum_{\substack{\lambda \\
\lambda_{1} \leq m}} q^{|\lambda|} P_{2 \lambda}^{\prime}\left(x_{1}^{ \pm}, \ldots, x_{n-1}^{ \pm}, 1 ; q\right) & \frac{1}{(q)_{\infty}^{n} \prod_{i=1}^{n-1}\left(q x_{i}^{ \pm}, q x_{i}^{ \pm 2}\right)_{\infty} \prod_{1 \leq i<j \leq n-1}\left(q x_{i}^{ \pm} x_{j}^{ \pm}\right)_{\infty}} \\
& \times \sum_{r_{1}, \ldots, r_{n-1}=-\infty}^{\infty} \sum_{r_{n}=0}^{\infty} \phi_{r_{n}} \frac{\Delta_{\mathrm{B}}\left(\hat{x} q^{r}\right)}{\Delta_{\mathrm{B}}(\hat{x})} \prod_{i=1}^{n} \hat{x}_{i}^{\kappa r_{i}} q^{\frac{1}{2} \kappa r_{i}^{2}-\frac{1}{2}(2 n-1) r_{i}} .
\end{aligned}
$$

It is easily checked that the summand on the right (without the factor $\phi_{r_{n}}$ ) is invariant under the variable change $r_{n} \mapsto-r_{n}$. Using the elementary relations

$$
\theta(-1 ; q)=2(-q)_{\infty}^{2}, \quad(-q)_{\infty}\left(q ; q^{2}\right)_{\infty}=1, \quad \theta(z,-z ; q) \theta\left(q z^{2} ; q^{2}\right)=\theta\left(z^{2}\right)
$$

we can then simplify the above to obtain

$$
\begin{aligned}
\sum_{\substack{\lambda \\
\lambda_{1} \leq m}} q^{|\lambda|} P_{2 \lambda}^{\prime}\left(x_{1}^{ \pm}, \ldots, x_{n-1}^{ \pm}, 1 ; q\right) & \frac{1}{(q)_{\infty}^{n} \prod_{i=1}^{n} \theta\left(\hat{x}_{i} ; q\right) \theta\left(q \hat{x}_{i}^{2} ; q^{2}\right) \prod_{1 \leq i<j \leq n} \theta\left(\hat{x}_{i} / \hat{x}_{j}, \hat{x}_{i} \hat{x}_{j} ; q\right)} \\
& \times \sum_{r \in \mathbb{Z}^{n}} \Delta_{\mathrm{B}}\left(\hat{x} q^{r}\right) \prod_{i=1}^{n} \hat{x}_{i}^{\kappa r_{i}-i+1} q^{\frac{1}{2} \kappa r_{i}^{2}-\frac{1}{2}(2 n-1) r_{i}} .
\end{aligned}
$$

The remainder of the proof is similar to that of (1.9). We make the simultaneous substitutions

$$
q \mapsto q^{2 n-1}, \quad x_{i} \mapsto q^{n-i}(1 \leq i \leq n)
$$


so that from here on $\hat{x}_{i}:=-q^{n-i}$. By the identity

$$
\begin{aligned}
\left(q^{2 n-1} ; q^{2 n-1}\right)_{\infty}^{n} \prod_{i=1}^{n} \theta\left(-q^{n-i} ; q^{2 n-1}\right) \theta\left(q^{2 n-2 i+1} ; q^{4 n-2}\right) & \\
& \times \prod_{1 \leq i<j \leq n} \theta\left(q^{j-i}, q^{2 n-i-j} ; q^{2 n-1}\right)=2(q)_{\infty}^{n}
\end{aligned}
$$

and (2.5), we find that

$$
\sum_{\substack{\lambda \\ \lambda_{1} \leq m}} q^{|\lambda|} P_{2 \lambda}\left(1, q, q^{2}, \ldots ; q^{2 n-1}\right)=\frac{\mathscr{M}}{2(q)_{\infty}^{n}}
$$

where we have that

$$
\mathscr{M}:=\sum_{r \in \mathbb{Z}^{n}} \Delta_{\mathrm{B}}\left(\hat{x} q^{(2 n-1) r}\right) \prod_{i=1}^{n} \hat{x}_{i}^{\kappa r_{i}-i+1} q^{\frac{1}{2}(2 n-1) \kappa r_{i}^{2}-\frac{1}{2}(2 n-1)^{2} r_{i}}
$$

By (3.14) and multilinearity, $\mathscr{M}$ can be rewritten in the form

$$
\mathscr{M}=\operatorname{det}_{1 \leq i, j \leq n}\left(\sum_{r \in \mathbb{Z}} \hat{x}_{i}^{\kappa r-i+1} q^{\frac{1}{2}(2 n-1) \kappa r^{2}-\frac{1}{2}(2 n-1)^{2} r} \cdot\left(\left(\hat{x}_{i} q^{(2 n-1) r}\right)^{j-1}-\left(\hat{x}_{i} q^{(2 n-1) r}\right)^{2 n-j}\right)\right)
$$

Following the same steps that led from (3.12) to (3.13), we obtain

$$
\begin{aligned}
& \mathscr{M}=\operatorname{det}_{1 \leq i, j \leq n}\left((-1)^{i-j} q^{b_{i j}} \sum_{r \in \mathbb{Z}}(-1)^{r} y_{i}^{(2 n-1) r-i+1} q^{(2 n-1) \kappa(}\left(\begin{array}{c}
r \\
2
\end{array}\right)\right. \\
&\left.\times\left(\left(y_{i} q^{\kappa r}\right)^{j-1}-\left(y_{i} q^{\kappa r}\right)^{2 n-j}\right)\right),
\end{aligned}
$$

where

$$
y_{i}=q^{\frac{1}{2}(\kappa+1)-i} \quad \text { and } \quad b_{i j}:=j^{2}-i^{2}+\frac{1}{2}(i-j)(\kappa+3) .
$$

Again, the factor $(-1)^{i-j} q^{b_{i j}}$ does not contribute, and so (3.14) then gives

$$
\mathscr{M}=\sum_{r \in \mathbb{Z}^{n}} \Delta_{\mathrm{B}}\left(y_{i} q^{\kappa r}\right) \prod_{i=1}^{n}(-1)^{r_{i}} y_{i}^{(2 n-1) r_{i}-i+1} q^{(2 n-1) \kappa\left(\begin{array}{c}
r_{i} \\
2
\end{array}\right)}
$$


To complete the proof, we apply the following variant of the $\mathrm{B}_{n}^{(1)}$ Macdonald identity 4

$$
\begin{aligned}
\sum_{r \in \mathbb{Z}^{n}} \Delta_{\mathrm{B}}\left(x q^{r}\right) \prod_{i=1}^{n}(-1)^{r_{i}} x_{i}^{(2 n-1) r_{i}-i+1} q^{(2 n-1)\left(\begin{array}{c}
r_{i} \\
2
\end{array}\right)} & \\
& =2(q)_{\infty}^{n} \prod_{i=1}^{n} \theta\left(x_{i} ; q\right) \prod_{1 \leq i<j \leq n} \theta\left(x_{i} / x_{j}, x_{i} x_{j} ; q\right),
\end{aligned}
$$

with $(q, x) \mapsto\left(q^{\kappa}, y\right)$.

Identity (1.7a) can be understood representation-theoretically, but this time the relevant Kac-Moody algebra is $\mathrm{A}_{2 n}^{(2)}$. According to [8, Lemma 2.3] the right-hand side of (3.24), with $\hat{x}$ interpreted (not as $\hat{x}=\left(-x_{1}, \ldots,-x_{n-1},-1\right)$ ) as

$$
\hat{x}_{i}=\mathrm{e}^{-\alpha_{0}-\cdots-\alpha_{n-i}} \quad(1 \leq i \leq n)
$$

and $q$ as

$$
q=\mathrm{e}^{-2 \alpha_{0}-\cdots-2 \alpha_{n-1}-\alpha_{n}},
$$

is the $\mathrm{A}_{2 n}^{(2)}$ character

$$
\mathrm{e}^{-m \Lambda_{n}} \operatorname{ch} V\left(m \Lambda_{n}\right)
$$

The substitution (3.25) corresponds to

$$
\mathrm{e}^{-\alpha_{0}} \mapsto-1 \quad \text { and } \quad \mathrm{e}^{-\alpha_{i}} \mapsto q(1 \leq i \leq n) .
$$

Denoting this by $F$, it is not hard to derive the general specialization formula $(3.31)$

$$
F\left(\mathrm{e}^{-\Lambda} \operatorname{ch} V(\Lambda)\right)=\frac{\left(q^{\kappa} ; q^{\kappa}\right)_{\infty}^{n}}{(q)_{\infty}^{n}} \prod_{i=1}^{n} \theta\left(q^{\lambda_{i}+n-i+1} ; q^{\kappa}\right) \prod_{1 \leq i<j \leq n} \theta\left(q^{\lambda_{i}-\lambda_{j}-i+j}, q^{\lambda_{i}+\lambda_{j}-i-j+2 n+2} ; q^{\kappa}\right)
$$

where $\Lambda$ is again parametrized as in (3.18), $\lambda_{0}-\lambda_{1}$ is even 5 , and $\kappa=2 n+\lambda_{0}+\lambda_{1}+1$. For $\lambda=\left(m^{n+1}\right)$ (so that $\left.\Lambda=m \Lambda_{n}\right)$ this yields the right-hand side of (1.7a).

3.4. Proof of Theorem $1.1(1.7 \mathrm{~b})$. Here we prove the companion result to (1.7a).

\footnotetext{
${ }^{4}$ The actual $\mathrm{B}_{n}^{(1)}$ Macdonald identity has the restriction $|r| \equiv 0(\bmod 2)$ in the sum over $r \in \mathbb{Z}^{n}$, which eliminates the factor 2 on the right. To prove the form used here it suffices to take the $a_{1}, \ldots, a_{2 n-1} \rightarrow 0$ and $a_{2 n} \rightarrow-1$ limit in Gustafson's multiple ${ }_{6} \psi_{6}$ summation for the affine root system $\mathrm{A}_{2 n-1}^{(2)}$, see [38].

${ }^{5}$ For $\lambda_{0}-\lambda_{1}$ odd, $F\left(\mathrm{e}^{-\Lambda} \operatorname{ch} V(\Lambda)\right)=0$.
} 
Proof of Theorem 1.1 (1.7b). In (3.21) we set $x_{n}=q^{1 / 2}$ so that

$$
\begin{aligned}
\sum_{\substack{\lambda \\
\lambda_{1} \leq m}} q^{|\lambda|} P_{2 \lambda}^{\prime}\left(x_{1}^{ \pm}, \ldots, x_{n-1}^{ \pm}, q^{1 / 2} ; q\right) & \frac{1}{(q)_{\infty}^{n-1}\left(q^{2}\right)_{\infty} \prod_{i=1}^{n-1}\left(q^{3 / 2} x_{i}^{ \pm}, q x_{i}^{ \pm 2}\right)_{\infty} \prod_{1 \leq i<j \leq n-1}\left(q x_{i}^{ \pm} x_{j}^{ \pm}\right)_{\infty}} \\
& \times \sum_{r_{1}, \ldots, r_{n-1}=-\infty}^{\infty} \sum_{r_{n}=0}^{\infty} \frac{\Delta_{\mathrm{C}}\left(\hat{x} q^{r}\right)}{\Delta_{\mathrm{C}}(\hat{x})} \prod_{i=1}^{n}(-1)^{r_{i}} \hat{x}_{i}^{\kappa r_{i}} q^{\frac{1}{2} \kappa r_{i}^{2}-n r_{i}},
\end{aligned}
$$

where $\kappa=2 m+2 n+1$ and $\hat{x}=\left(x_{1}, \ldots, x_{n-1}, q^{1 / 2}\right)$. The $r_{n}$-dependent part of the summand is

$$
(-1)^{r_{n}} q^{\kappa\left(\begin{array}{c}
r_{n}+1 \\
2
\end{array}\right)-n r_{n}} \frac{1-q^{2 r_{n}+1}}{1-q} \prod_{i=1}^{n-1} \frac{x_{i} q^{r_{i}}-q^{r_{n}+1 / 2}}{x_{i}-q^{1 / 2}} \cdot \frac{x_{i} q^{r_{n}+r_{i}+1 / 2}-1}{x_{i} q^{1 / 2}-1}
$$

which is readily checked to be invariant under the substitution $r_{n} \mapsto-r_{n}-1$. Hence

$$
\begin{aligned}
\sum_{\substack{\lambda \\
\lambda_{1} \leq m}} q^{|\lambda|} & P_{2 \lambda}^{\prime}\left(x_{1}^{ \pm}, \ldots, x_{n-1}^{ \pm}, q^{1 / 2} ; q\right) \\
= & \frac{1}{2(q)_{\infty}^{n} \prod_{i=1}^{n-1}(-1) \theta\left(q^{1 / 2} x_{i}, x_{i}^{2} ; q\right) \prod_{1 \leq i<j \leq n-1} \theta\left(x_{i} / x_{j}, x_{i} x_{j} ; q\right)} \\
& \quad \times \sum_{r \in \mathbb{Z}^{n}} \Delta_{\mathrm{C}}\left(\hat{x} q^{r}\right) \prod_{i=1}^{n}(-1)^{r_{i}} \hat{x}_{i}^{\kappa r_{i}-i} q^{\frac{1}{2} \kappa r_{i}^{2}-n r_{i}+\frac{1}{2}} .
\end{aligned}
$$

Our next step is to replace $x_{i} \mapsto x_{n-i+1}$ and $r_{i} \mapsto r_{n-i+1}$. By $\theta(x ; q)=-x \theta\left(x^{-1} ; q\right)$ and (3.23), this leads to

$$
\begin{aligned}
\sum_{\substack{\lambda \\
\lambda_{1} \leq m}} q^{|\lambda|} & P_{2 \lambda}^{\prime}\left(q^{1 / 2}, x_{2}^{ \pm}, \ldots, x_{n}^{ \pm} ; q\right) \\
= & \frac{1}{(q)_{\infty}^{n} \prod_{i=1}^{n} \theta\left(-q^{1 / 2} \hat{x}_{i} ; q\right) \theta\left(\hat{x}_{i}^{2} ; q^{2}\right) \prod_{1 \leq i<j \leq n} \theta\left(\hat{x}_{i} / \hat{x}_{j}, \hat{x}_{i} \hat{x}_{j} ; q\right)} \\
& \quad \times \sum_{r \in \mathbb{Z}^{n}} \Delta_{\mathrm{C}}\left(\hat{x} q^{r}\right) \prod_{i=1}^{n}(-1)^{r_{i}} \hat{x}_{i}^{\kappa r_{i}-i+1} q^{\frac{1}{2} \kappa r_{i}^{2}-n r_{i}}
\end{aligned}
$$

where now $\hat{x}=\left(q^{1 / 2}, x_{2}, \ldots, x_{n}\right)$. Again we are at the point where we can specialize, letting

$$
q \mapsto q^{2 n-1}, \quad x_{i} \mapsto q^{n-i+1 / 2}=: \hat{x}_{i} \quad(1 \leq i \leq n)
$$


This is consistent, since $x_{1}=q^{1 / 2} \mapsto q^{n-1 / 2}$. By the identity

$$
\begin{aligned}
\left(q^{2 n-1} ; q^{2 n-1}\right)_{\infty}^{n} \prod_{i=1}^{n} \theta\left(-q^{2 n-i} ; q^{2 n-1}\right) \theta\left(q^{2 n-2 i+1} ; q^{4 n-2}\right) & \\
& \times \prod_{1 \leq i<j \leq n} \theta\left(q^{j-i}, q^{2 n-i-j+1} ; q^{2 n-1}\right)=2(q)_{\infty}^{n},
\end{aligned}
$$

we obtain

$$
\sum_{\substack{\lambda \\ \lambda_{1} \leq m}} q^{2|\lambda|} P_{2 \lambda}\left(1, q, q^{2}, \ldots ; q^{2 n-1}\right)=\frac{\mathscr{M}}{2(q)_{\infty}^{n}}
$$

where

$$
\mathscr{M}:=\sum_{r \in \mathbb{Z}^{n}} \Delta_{\mathrm{C}}\left(\hat{x} q^{(2 n-1) r}\right) \prod_{i=1}^{n}(-1)^{r_{i}} \hat{x}_{i}^{\kappa r_{i}-i+1} q^{\frac{1}{2}(2 n-1) \kappa r_{i}^{2}-(2 n-1) n r_{i}} .
$$

Expressing $\mathscr{M}$ in determinantal form using (3.11) yields

$$
\begin{aligned}
\mathscr{M}=\operatorname{det}_{1 \leq i, j \leq n}\left(\sum_{r \in \mathbb{Z}}(-1)^{r} \hat{x}_{i}^{\kappa r-i+1} q^{\frac{1}{2}(2 n-1) \kappa r^{2}-(2 n-1) n r}\right. & \\
& \left.\times\left(\left(\hat{x}_{i} q^{(2 n-1) r}\right)^{j-1}-\left(\hat{x}_{i} q^{(2 n-1) r}\right)^{2 n-j+1}\right)\right) .
\end{aligned}
$$

We now replace $(i, j) \mapsto(j, i)$ and, viewing the resulting determinant as of the form $\operatorname{det}\left(\sum_{r} u_{i j ; r}-\sum_{r} v_{i j ; r}\right)$, we change the summation index $r \mapsto-r$ in the sum over $u_{i j ; r}$. The expression for $\mathscr{M}$ we obtain is exactly (3.26) except that $(-1)^{i-j} q^{b_{i j}}$ is replaced by $q^{c_{i j}}$ and $y_{i}$ is given by $q^{n-i+1}$ instead of $q^{(\kappa+1) / 2-i}$. Following the previous proof results in (1.7b).

To interpret (1.7b) in terms of $\mathrm{A}_{2 n}^{(2)}$, we note that by [8, Lemma 2.2] the right-hand side of (3.32) in which $\hat{x}$ is interpreted as

$$
\hat{x}_{i}=-q^{1 / 2} \mathrm{e}^{\alpha_{0}+\cdots+\alpha_{i-1}} \quad(1 \leq i \leq n)
$$

(and $q$ again as (3.29) ) corresponds to the $\mathrm{A}_{2 n}^{(2)}$ character

$$
\mathrm{e}^{-2 m \Lambda_{0}} \operatorname{ch} V\left(2 m \Lambda_{0}\right)
$$

The specialization (3.33) is then again consistent with (3.30). From (3.31) with $\lambda=$ $\left(2 m, 0^{n}\right)$, the first product-form on the right of (1.7b) immediately follows. By level-rank duality, we can also identify (1.7b) as a specialization of the $\mathrm{A}_{2 m}^{(2)} \operatorname{character} \mathrm{e}^{-2 n \Lambda_{0}} \operatorname{ch} V\left(2 n \Lambda_{0}\right)$. 
3.5. Proof of Theorem 1.3. This proof, which uses the $\mathrm{D}_{n}^{(1)}$ Macdonald identity, is the most complicated of the four.

Proof of Theorem 1.3. Once again we iterate (3.7), but now both $x_{n-1}$ and $x_{n}$ remain unpaired:

$$
\lim _{y_{1} \rightarrow x_{1}^{-1}} \ldots \lim _{y_{n-2} \rightarrow x_{n-2}^{-1}} L_{m}^{(0)}\left(x_{1}, y_{1}, \ldots, x_{n-2}, y_{n-2}, x_{n-1}, x_{n}\right)=L_{m}^{(n-2)}\left(x_{1}, \ldots, x_{n}\right)
$$

Accordingly, if we replace $x \mapsto\left(x_{1}, y_{1}, \ldots, x_{n-2}, y_{n-2}, x_{n-1}, x_{n}\right)$ in (3.3) (thereby changing the rank from $n$ to $2 n-2)$ and take the $y_{i} \rightarrow x_{i}^{-1}$ limit, for $1 \leq i \leq n-2$, we obtain

$$
\begin{aligned}
& \sum_{\substack{\lambda \\
\lambda_{1} \leq m}} q^{|\lambda|} P_{2 \lambda}^{\prime}\left(x_{1}^{ \pm}, \ldots, x_{n-2}^{ \pm}, x_{n-1}, x_{n} ; q\right) \\
& =\frac{1}{(q)_{\infty}^{n-2}\left(q x_{n-1}^{2}, q x_{n-1} x_{n}, q x_{n}^{2}\right)_{\infty}} \\
& \quad \times \frac{1}{\prod_{i=1}^{n-2}\left(q x_{i}^{ \pm 2}, q x_{i}^{ \pm} x_{n-1}, q x_{i}^{ \pm} x_{n}\right)_{\infty} \prod_{1 \leq i<j \leq n-2}\left(q x_{i}^{ \pm} x_{j}^{ \pm}\right)_{\infty}} \\
& \quad \times \sum_{r \in \mathbb{Z}^{n}} \frac{\Delta_{\mathrm{C}}\left(x q^{r}\right)}{\Delta_{\mathrm{C}}(x)} \prod_{i=1}^{n}\left(\frac{x_{i}^{\kappa}}{x_{n-1} x_{n}}\right)^{r_{i}} q^{\frac{1}{2} \kappa r_{i}^{2}-(n-1) r_{i}} \frac{\left(x_{i} x_{n-1}, x_{i} x_{n}\right)_{r_{i}}}{\left(q x_{i} / x_{n-1}, q x_{i} / x_{n}\right)_{r_{i}}}
\end{aligned}
$$

where $\kappa=2 m+2 n$. It is important to note that the summand vanishes unless $r_{n-1}$ and $r_{n}$ are both nonnegative. Next we let $\left(x_{n-1}, x_{n}\right)$ tend to $\left(q^{1 / 2}, 1\right)$ using

$$
\lim _{\left(x_{n-1}, x_{n}\right) \rightarrow\left(q^{1 / 2}, 1\right)} \frac{\Delta_{\mathrm{C}}\left(x q^{r}\right)}{\Delta_{\mathrm{C}}(x)} \prod_{i=1}^{n} \frac{\left(x_{i} x_{n-1}, x_{i} x_{n}\right)_{r_{i}}}{\left(q x_{i} / x_{n-1}, q x_{i} / x_{n}\right)_{r_{i}}}=\phi_{r_{n}} \frac{\Delta_{\mathrm{B}}\left(\hat{x} q^{r}\right)}{\Delta_{\mathrm{B}}(\hat{x})}
$$

with $\phi_{r}$ as in (3.22) and $\hat{x}:=\left(-x_{1}, \ldots,-x_{n-2},-q^{1 / 2},-1\right)$. Hence we find that

$$
\begin{aligned}
& \sum_{\substack{\lambda \\
\lambda_{1} \leq m}} q^{|\lambda|} P_{2 \lambda}^{\prime}\left(x_{1}^{ \pm}, \ldots, x_{n-2}^{ \pm}, q^{1 / 2}, 1 ; q\right) \\
& =\frac{1}{(q)_{\infty}^{n-1}\left(q^{3 / 2} ; q^{1 / 2}\right)_{\infty} \prod_{i=1}^{n-2}\left(q x_{i}^{ \pm} ; q^{1 / 2}\right)_{\infty}\left(q x_{i}^{ \pm 2}\right)_{\infty} \prod_{1 \leq i<j \leq n-2}\left(q x_{i}^{ \pm} x_{j}^{ \pm}\right)_{\infty}} \\
& \quad \times \sum_{r_{1}, \ldots, r_{n-2}=-\infty}^{\infty} \sum_{r_{n-1}, r_{n}=0}^{\infty} \phi_{r_{n}} \frac{\Delta_{\mathrm{B}}\left(\hat{x} q^{r}\right)}{\Delta_{\mathrm{B}}(\hat{x})} \prod_{i=1}^{n} \hat{x}_{i}^{\kappa r_{i}} q^{\frac{1}{2} \kappa r_{i}^{2}-\frac{1}{2}(2 n-1) r_{i}} .
\end{aligned}
$$


Since the summand (without the factor $\phi_{r_{n}}$ ) is invariant under the variable change $r_{n} \mapsto$ $-r_{n}$, as well as the change $r_{n-1} \mapsto-r_{n-1}-1$, we can rewrite this as

$$
\begin{aligned}
\sum_{\substack{\lambda \\
\lambda_{1} \leq m}} q^{|\lambda|} & P_{2 \lambda}^{\prime}\left(x_{1}^{ \pm}, \ldots, x_{n-2}^{ \pm}, q^{1 / 2}, 1 ; q\right) \\
= & \frac{1}{(q)_{\infty}^{n-1}\left(q^{1 / 2} ; q^{1 / 2}\right)_{\infty} \prod_{i=1}^{n} \theta\left(\hat{x}_{i} ; q^{1 / 2}\right) \prod_{1 \leq i<j \leq n} \theta\left(\hat{x}_{i} / \hat{x}_{j}, \hat{x}_{i} \hat{x}_{j}\right)} \\
& \times \sum_{r \in \mathbb{Z}^{n}} \Delta_{\mathrm{B}}\left(\hat{x} q^{r}\right) \prod_{i=1}^{n} \hat{x}_{i}^{\kappa r_{i}-i+1} q^{\frac{1}{2} \kappa r_{i}^{2}-\frac{1}{2}(2 n-1) r_{i}},
\end{aligned}
$$

where, once again, we have used (3.23) to clean up the infinite products. Before we can carry out the usual specialization, we need to relabel $x_{1}, \ldots, x_{n-2}$ as $x_{2}, \ldots, x_{n-1}$ and, accordingly, we redefine $\hat{x}$ as $\left(-q^{1 / 2},-x_{2}, \ldots,-x_{n-1},-1\right)$. For $n \geq 2$, we then find that

$$
\begin{aligned}
\sum_{\substack{\lambda \\
\lambda_{1} \leq m}} q^{|\lambda|} P_{2 \lambda}^{\prime}\left(q^{1 / 2}, x_{2}^{ \pm}, \ldots, x_{n-1}^{ \pm}, 1 ; q\right) & \frac{1}{=} \frac{1}{(q)_{\infty}^{n-1}\left(q^{1 / 2} ; q^{1 / 2}\right)_{\infty} \prod_{i=1}^{n} \theta\left(\hat{x}_{i} ; q^{1 / 2}\right) \prod_{1 \leq i<j \leq n} \theta\left(\hat{x}_{i} / \hat{x}_{j}, \hat{x}_{i} \hat{x}_{j}\right)} \\
& \times \sum_{r \in \mathbb{Z}^{n}} \Delta_{\mathrm{B}}\left(\hat{x} q^{r}\right) \prod_{i=1}^{n} \hat{x}_{i}^{\kappa r_{i}-i+1} q^{\frac{1}{2} \kappa r_{i}^{2}-\frac{1}{2}(2 n-1) r_{i}} .
\end{aligned}
$$

We are now ready to make the substitutions

$$
q \mapsto q^{2 n-2}, \quad x_{i} \mapsto q^{n-i}(2 \leq i \leq n-1),
$$

so that $\hat{x}_{i}:=-q^{n-i}$ for $1 \leq i \leq n$. By the identity

$$
\begin{aligned}
\left(q^{2 n-2} ; q^{2 n-2}\right)_{\infty}^{n-1}\left(q^{n-1} ; q^{n-1}\right)_{\infty} \prod_{i=1}^{n} \theta( & \left.-q^{n-i} ; q^{n-1}\right) \\
& \times \prod_{1 \leq i<j \leq n} \theta\left(q^{j-i}, q^{2 n-i-j} ; q^{2 n-2}\right)=4\left(q^{2} ; q^{2}\right)_{\infty}(q)_{\infty}^{n-1}
\end{aligned}
$$

and (2.5), we obtain

$$
\sum_{\substack{\lambda \\ \lambda_{1} \leq m}} q^{2|\lambda|} P_{2 \lambda}\left(1, q, q^{2}, \ldots ; q^{2 n-3}\right)=\frac{\mathscr{M}}{4\left(q^{2} ; q^{2}\right)_{\infty}(q)_{\infty}^{n-1}},
$$

where $\mathscr{M}$ is given by

$$
\mathscr{M}:=\sum_{r \in \mathbb{Z}^{n}} \Delta_{\mathrm{B}}\left(\hat{x} q^{2(n-1) r}\right) \prod_{i=1}^{n} \hat{x}_{i}^{\kappa r_{i}-i+1} q^{(n-1) \kappa r_{i}^{2}-(n-1)(2 n-1) r_{i}}
$$


By the $\mathrm{B}_{n}$ determinant (3.14), we find that

$$
\mathscr{M}=\operatorname{det}_{1 \leq i, j \leq n}\left(\sum_{r \in \mathbb{Z}} \hat{x}_{i}^{\kappa r-i+1} q^{(n-1) \kappa r^{2}-(n-1)(2 n-1) r} \cdot\left(\left(\hat{x}_{i} q^{2(n-1) r}\right)^{j-1}-\left(\hat{x}_{i} q^{2(n-1) r}\right)^{2 n-j}\right)\right) .
$$

By the same substitutions that transformed (3.12) into (3.13), we obtain

$$
\mathscr{M}=\operatorname{det}_{1 \leq i, j \leq n}\left((-1)^{i-j} q^{b_{i j}} \sum_{r \in \mathbb{Z}} y_{i}^{2(n-1) r-i+1} q^{2(n-1) \kappa\left(\begin{array}{c}
r \\
2
\end{array}\right)} \cdot\left(\left(y_{i} q^{\kappa r}\right)^{j-1}+\left(y_{i} q^{\kappa r}\right)^{2 n-j-1}\right)\right),
$$

where $y_{i}$ and $b_{i j}$ are as in (3.27). Recalling the Weyl denominator formula for $\mathrm{D}_{n}$ [46]

$$
\frac{1}{2} \operatorname{det}_{1 \leq i, j \leq n}\left(x_{i}^{j-1}+x_{i}^{2 n-j-1}\right)=\prod_{1 \leq i<j \leq n}\left(x_{i}-x_{j}\right)\left(x_{i} x_{j}-1\right)=: \Delta_{\mathrm{D}}(x)
$$

we can rewrite $\mathscr{M}$ in the form

$$
\mathscr{M}=2 \sum_{r \in \mathbb{Z}^{n}} \Delta_{\mathrm{D}}\left(x q^{r}\right) \prod_{i=1}^{n} y_{i}^{2(n-1) r_{i}-i+1} q^{2(n-1) \kappa\left(\begin{array}{c}
r_{i} \\
2
\end{array}\right)}
$$

Taking the $a_{1}, \ldots, a_{2 n-2} \rightarrow 0, a_{2 n-1} \rightarrow 1$ and $a_{2 n} \rightarrow-1$ limit in Gustafson's multiple ${ }_{6} \psi_{6}$ summation for the affine root system $\mathrm{A}_{2 n-1}^{(2)}$ [38] leads to the following variant of the $\mathrm{D}_{n}^{(1)}$ Macdonald identity 6

$$
\sum_{r \in \mathbb{Z}^{n}} \Delta_{\mathrm{D}}\left(x q^{r}\right) \prod_{i=1}^{n} x_{i}^{2(n-1) r_{i}-i+1} q^{2(n-1)\left(\begin{array}{c}
r_{i} \\
2
\end{array}\right)}=2(q)_{\infty}^{n} \prod_{1 \leq i<j \leq n} \theta\left(x_{i} / x_{j}, x_{i} x_{j} ; q\right) .
$$

This implies the claimed product form for $\mathscr{M}$ and completes our proof.

Identity (1.10) has a representation-theoretic interpretation. By [8, Lemma 2.4], the right-hand side of (3.34) in which $\hat{x}$ is interpreted as

$$
\hat{x}_{i}=\mathrm{e}^{-\alpha_{i}-\cdots-\alpha_{n}} \quad(1 \leq i \leq n)
$$

and $q$ as

yields the $\mathrm{D}_{n+1}^{(2)}$ character

$$
q=\mathrm{e}^{-2 \alpha_{0}-\cdots-2 \alpha_{n}}
$$

$$
\mathrm{e}^{-2 m \Lambda_{0}} \operatorname{ch} V\left(2 m \Lambda_{0}\right)
$$

The specialization (3.35) then corresponds to

$$
\mathrm{e}^{-\alpha_{0}}, \mathrm{e}^{-\alpha_{n}} \mapsto-1 \quad \text { and } \quad \mathrm{e}^{-\alpha_{i}} \mapsto q(2 \leq i \leq n-1) .
$$

Denoting this by $F$, we have

$$
F\left(\mathrm{e}^{-\Lambda} \operatorname{ch} V(\Lambda)\right)=\frac{\left(q^{\kappa} ; q^{\kappa}\right)_{\infty}^{n}}{\left(q^{2} ; q^{2}\right)_{\infty}(q)_{\infty}^{n-1}} \prod_{1 \leq i<j \leq n} \theta\left(q^{\lambda_{i}-\lambda_{j}-i+j}, q^{\lambda_{i}+\lambda_{j}-i-j+2 n+1} ; q^{\kappa}\right)
$$

\footnotetext{
${ }^{6} \mathrm{As}$ in the $\mathrm{B}_{n}^{(1)}$ case, the actual $\mathrm{D}_{n}^{(1)}$ Macdonald identity contains the restriction $|r| \equiv 0(\bmod 2)$ on the sum over $r$.
} 
where $\kappa=2 n+2 \lambda_{0}$ and

$$
\Lambda=2\left(\lambda_{0}-\lambda_{1}\right) \Lambda_{0}+\left(\lambda_{1}-\lambda_{2}\right) \Lambda_{1}+\cdots+\left(\lambda_{n-1}-\lambda_{n}\right) \Lambda_{n-1}+2 \lambda_{n} \Lambda_{n}
$$

for $\lambda=\left(\lambda_{0}, \lambda_{1}, \ldots, \lambda_{n}\right)$ a partition or half-partition (i.e., all $\left.\lambda_{i} \in \mathbb{Z}+1 / 2\right)$. For $\lambda=\left(m, 0^{n}\right)$ this agrees with (1.10).

\section{Proof of Theorem 1.5}

For integers $k$ and $m$, where $0 \leq k \leq m$, we denote the the nearly-rectangular partition $(\underbrace{m, \ldots, m}_{r \text { times }}, k)$ as $\left(m^{r}, k\right)$. Using these partitions, we have the following "limiting" RogersRamanujan-type identities, which imply Theorem 1.5 when $k=0$ or $k=m$.

Theorem 4.1 ( $\mathrm{A}_{n-1}^{(1)} \mathrm{RR}$ and $\mathrm{AG}$ identities). If $m$ and $n$ are positive integers and $0 \leq$ $k \leq m$, then we have

$$
\begin{aligned}
\lim _{r \rightarrow \infty} q^{-m\left(\begin{array}{c}
r \\
2
\end{array}\right)-k r} Q_{\left(m^{r}, k\right)} & \left(1, q, q^{2}, \ldots ; q^{n}\right) \\
= & \frac{\left(q^{n} ; q^{n}\right)_{\infty}\left(q^{\kappa} ; q^{\kappa}\right)_{\infty}^{n-1}}{(q)_{\infty}^{n}} \cdot \prod_{i=1}^{n-1} \theta\left(q^{i+k} ; q^{\kappa}\right) \cdot \prod_{1 \leq i<j \leq n-1} \theta\left(q^{j-i} ; q^{\kappa}\right),
\end{aligned}
$$

where $\kappa=m+n$.

Remark. A similar calculation when $k \geq m$ gives

$$
\begin{aligned}
\lim _{r \rightarrow \infty} q^{-m\left(\begin{array}{c}
r+1 \\
2
\end{array}\right)} Q_{\left(k, m^{r}\right)}\left(1, q, q^{2}\right. & \left.\ldots ; q^{n}\right) \\
& =\left[\begin{array}{c}
k-m+n-1 \\
n-1
\end{array}\right]_{q} \frac{\left(q^{n} ; q^{n}\right)_{\infty}\left(q^{\kappa} ; q^{\kappa}\right)_{\infty}^{n-1}}{(q)_{\infty}^{n}} \prod_{1 \leq i<j \leq n} \theta\left(q^{j-i} ; q^{\kappa}\right) .
\end{aligned}
$$

Proof of Theorem 4.1. It suffices to prove the identity for $0 \leq k<m$, and below we assume that $k$ satisfies this inequality.

The following identity for modified Hall-Littlewood polynomials indexed by near-rectangular partitions is a special case of [8, Corollary 3.2]:

$$
\begin{aligned}
Q_{\left(m^{r}, k\right)}^{\prime}(x ; q)=(q)_{r}(q)_{1} \sum_{\substack{u \in \mathbb{Z}_{+}^{n} \\
|u|=r+1}} \sum_{\substack{v \in \mathbb{Z}_{+}^{n} \\
|v|=r}} \prod_{i=1}^{n} x_{i}^{k u_{i}+(m-k) v_{i}} q^{k\left(\begin{array}{c}
u_{i} \\
2
\end{array}\right)+(m-k)\left(\begin{array}{c}
v_{i} \\
2
\end{array}\right)} & \\
& \times \prod_{i, j=1}^{n} \frac{\left(q x_{i} / x_{j}\right)_{u_{i}-u_{j}}}{\left(q x_{i} / x_{j}\right)_{u_{i}-v_{j}}} \cdot \frac{\left(q x_{i} / x_{j}\right)_{v_{i}-v_{j}}}{\left(q x_{i} / x_{j}\right)_{v_{i}}} .
\end{aligned}
$$

It is enough to compute the limit on the left-hand side of (4.1) for $r$ a multiple of $n$. Hence we replace $r$ by $n r$ in the above expression, and then shift $u_{i} \mapsto u_{i}+r$ and $v_{i} \mapsto v_{i}+r$, 
for all $1 \leq i \leq n$, to obtain

$$
\begin{aligned}
Q_{\left(m^{n r}, k\right)}^{\prime}(x ; q) & =\left(x_{1} \cdots x_{n}\right)^{m r} q^{m n\left(\begin{array}{c}
r \\
2
\end{array}\right)+k r}(q)_{n r}(q)_{1} \\
& \times \sum_{\substack{u \in \mathbb{Z}^{n} \\
|u|=1}} \sum_{\substack{v \in \mathbb{Z}^{n} \\
|v|=0}} \prod_{i=1}^{n} x_{i}^{k u_{i}+(m-k) v_{i}} q^{k\left(\begin{array}{c}
u_{i} \\
2
\end{array}\right)+(m-k)\left(\begin{array}{c}
v_{i} \\
2
\end{array}\right)} \prod_{i, j=1}^{n} \frac{\left(q x_{i} / x_{j}\right)_{u_{i}-u_{j}}}{\left(q x_{i} / x_{j}\right)_{u_{i}-v_{j}}} \cdot \frac{\left(q x_{i} / x_{j}\right)_{v_{i}-v_{j}}}{\left(q x_{i} / x_{j}\right)_{r+v_{i}}} .
\end{aligned}
$$

Since the summand vanishes unless $u_{i} \geq v_{i}$ for all $i$ and $|u|=|v|+1$, it follows that $u=v+\epsilon_{\ell}$, for some $\ell=1, \ldots, n$, where $\left(\epsilon_{\ell}\right)_{i}=\delta_{\ell i}$. Hence we find that

$$
\begin{aligned}
Q_{\left(m^{n r}, k\right)}^{\prime}(x ; q)= & \left(x_{1} \cdots x_{n}\right)^{m r} q^{m n\left(\begin{array}{c}
r \\
2
\end{array}\right)+k r}(q)_{n r} \\
& \times \sum_{\substack{v \in \mathbb{Z}^{n} \\
|v|=0}} \prod_{i=1}^{n} x_{i}^{m v_{i}} q^{m\left(\begin{array}{c}
v_{i} \\
2
\end{array}\right)} \prod_{i, j=1}^{n} \frac{\left(q x_{i} / x_{j}\right)_{v_{i}-v_{j}}}{\left(q x_{i} / x_{j}\right)_{r+v_{i}}} \sum_{\ell=1}^{n}\left(x_{\ell} q^{v_{\ell}}\right)^{k} \prod_{\substack{i=1 \\
i \neq k}}^{n} \frac{1}{1-q^{v_{i}-v_{\ell}} x_{i} / x_{\ell}} .
\end{aligned}
$$

Next we use

$$
\prod_{i, j=1}^{n}\left(q x_{i} / x_{j}\right)_{v_{i}-v_{j}}=\frac{\Delta\left(x q^{v}\right)}{\Delta(x)}(-1)^{(n-1)|v|} q^{-\left(\begin{array}{c}
|v| \\
2
\end{array}\right)} \prod_{i=1}^{n} x_{i}^{n v_{i}-|v|} q^{n\left(\begin{array}{c}
v_{i} \\
2
\end{array}\right)+(i-1) v_{i}},
$$

where $\Delta(x):=\prod_{1 \leq i<j \leq n}\left(1-x_{i} / x_{j}\right)$, and

$$
\sum_{\ell=1}^{n} x_{\ell}^{k} \prod_{\substack{i=1 \\ i \neq k}}^{n} \frac{1}{1-x_{i} / x_{\ell}}=\sum_{1 \leq i_{1} \leq i_{2} \leq \cdots \leq i_{k} \leq n} x_{i_{1}} x_{i_{2}} \cdots x_{i_{k}}=h_{k}(x)=s_{(k)}(x),
$$

where $h_{k}$ and $s_{\lambda}$ are the complete symmetric and Schur function, respectively. Thus we have

$$
\begin{aligned}
& Q_{\left(m^{n r}, k\right)}^{\prime}(x ; q)=\left(x_{1} \cdots x_{n}\right)^{m r} q^{m n\left(\begin{array}{c}
r \\
2
\end{array}\right)+k r}(q)_{n r} \\
& \times \sum_{\substack{v \in \mathbb{Z}^{n} \\
|v|=0}} s_{(k)}\left(x q^{v}\right) \frac{\Delta\left(x q^{v}\right)}{\Delta(x)} \prod_{i=1}^{n} x_{i}^{\kappa v_{i}} q^{\frac{1}{2} \kappa v_{i}^{2}+i v_{i}} \prod_{i, j=1}^{n} \frac{1}{\left(q x_{i} / x_{j}\right)_{r+v_{i}}},
\end{aligned}
$$

where $\kappa:=m+n$. Note that the summand vanishes unless $v_{i} \geq-r$ for all $i$. This implies the limit

$$
\begin{aligned}
\lim _{r \rightarrow \infty} q^{-m n\left(\begin{array}{c}
r \\
2
\end{array}\right)-k r} \frac{Q_{\left(m^{n r}, k\right)}^{\prime}(x ; q)}{\left(x_{1} \cdots x_{n}\right)^{m r}} & \\
& =\frac{1}{(q)_{\infty}^{n-1} \prod_{1 \leq i<j \leq n} \theta\left(x_{i} / x_{j} ; q\right)} \sum_{\substack{v \in \mathbb{Z}^{n} \\
|v|=0}} s_{(k)}\left(x q^{v}\right) \Delta\left(x q^{v}\right) \prod_{i=1}^{n} x_{i}^{\kappa v_{i}} q^{\frac{1}{2} \kappa v_{i}^{2}+i v_{i}} .
\end{aligned}
$$

The expression on the right is exactly the Weyl-Kac formula for the level- $m \mathrm{~A}_{n-1}^{(1)}$ character [43]

$$
\mathrm{e}^{-\Lambda} \operatorname{ch} V(\Lambda), \quad \Lambda=(m-k) \Lambda_{0}+k \Lambda_{1}
$$


provided we identify

$$
q=\mathrm{e}^{-\alpha_{0}-\alpha_{1}-\cdots-\alpha_{n-1}} \quad \text { and } \quad x_{i} / x_{i+1}=\mathrm{e}^{-\alpha_{i}}(1 \leq i \leq n-1) .
$$

Hence

$$
\lim _{r \rightarrow \infty} q^{-m n\left(\begin{array}{c}
r \\
2
\end{array}\right)-k r} \frac{Q_{\left(m^{n r}, k\right)}^{\prime}(x ; q)}{\left(x_{1} \cdots x_{n}\right)^{m r}}=\mathrm{e}^{-\Lambda} \operatorname{ch} V(\Lambda),
$$

with $\Lambda$ as above. For $m=1$ and $k=0$ this was obtained in [45] by more elementary means. The simultaneous substitutions $q \mapsto q^{n}$ and $x_{i} \mapsto q^{n-i}$ correspond to the principal specialization (3.16). From (3.17) we can then read off the product form claimed in (4.1).

\section{Siegel Functions}

The normalizations for the series $\Phi_{*}$ were chosen so that the resulting $q$-series are modular functions on the congruence subgroups $\Gamma(N)$, where

$$
\Gamma(N):=\left\{\left(\begin{array}{ll}
a & b \\
c & d
\end{array}\right) \in \mathrm{SL}_{2}(\mathbb{Z}): a \equiv d \equiv 1 \quad(\bmod N), b \equiv c \equiv 0 \quad(\bmod N)\right\} .
$$

These groups act on $\mathbb{H}$, the upper-half of the complex plane, by $\gamma \tau:=\frac{a \tau+b}{c \tau+d}$, where $\gamma=$ $\left(\begin{array}{ll}a & b \\ c & d\end{array}\right)$. If $f$ is a meromorphic function on $\mathbb{H}$ and $\gamma \in \mathrm{SL}_{2}(Z)$, then we define

$$
\left(\left.f\right|_{k} \gamma\right)(\tau):=(c \tau+d)^{-k} f(\gamma \tau)
$$

Modular functions are meromorphic functions which are invariant with respect to this action. More precisely, a meromorphic function $f$ on $\mathbb{H}$ is a modular function on $\Gamma(N)$ if for every $\gamma \in \Gamma(N)$ we have

$$
f(\gamma \tau)=\left(\left.f\right|_{0} \gamma\right)(\tau)=f(\tau)
$$

The set of such functions forms a field. We let $\mathcal{F}_{N}$ denote the canonical subfield of those modular functions on $\Gamma(N)$ whose Fourier expansions are defined over $\mathbb{Q}\left(\zeta_{N}\right)$, where $\zeta_{N}:=$ $\mathrm{e}^{2 \pi \mathrm{i} / N}$.

The important work of Kubert and Lang [47] plays a central role in the study of these modular function fields. Their work, which is built around the Siegel $g_{a}$ functions and the Klein $\mathfrak{t}_{a}$ functions, allows us to understand the fields $\mathcal{F}_{N}$, as well as the Galois theoretic properties of the extensions $\mathcal{F}_{N} / \mathcal{F}_{1}$. These results will be fundamental tools in the proofs of Theorems 1.6 and 1.7 .

5.1. Basic Facts about Siegel functions. We begin by recalling the definitions of the Siegel and Klein functions. Let $\mathbf{B}_{2}(x):=x^{2}-x+\frac{1}{6}$ be the second Bernoulli polynomial and $\mathrm{e}(x):=\mathrm{e}^{2 \pi \mathrm{i} x}$. If $a=\left(a_{1}, a_{2}\right) \in \mathbb{Q}^{2}$, then the Siegel function $g_{a}$ is defined as

$$
g_{a}(\tau):=q^{\frac{1}{2} \mathbf{B}_{2}\left(a_{1}\right)} \mathrm{e}\left(a_{2}\left(a_{1}-1\right) / 2\right) \prod_{n=1}^{\infty}\left(1-q^{n-1+a_{1}} \mathrm{e}\left(a_{2}\right)\right)\left(1-q^{n-a_{1}} \mathrm{e}\left(-a_{2}\right)\right) .
$$


Notice this is a change of sign from the usual normalization of the Siegel function. The Klein function $\mathfrak{t}_{a}$ is defined as

$$
\mathfrak{t}_{a}(\tau):=-\frac{g_{a}(\tau)}{\eta(\tau)^{2}}
$$

where $\eta(\tau):=q^{1 / 24} \prod_{n=1}^{\infty}\left(1-q^{n}\right)$ is the Dedekind $\eta$-function.

Neither $g_{a}$ nor $\mathfrak{t}_{a}$ are modular on $\Gamma(N)$, however if $N \cdot a \in \mathbb{Z}^{2}$, then $\mathfrak{t}_{a}^{2 N}$ is on $\Gamma(N)$ (or $\mathfrak{t}_{a}^{N}$ if $N$ is odd). Therefore if $g_{a}^{\operatorname{lcm}(12,2 N)} \in \mathcal{F}_{N}$, and if $N \cdot a^{\prime} \in \mathbb{Z}^{2}$, then $\left(\frac{g_{a}(\tau)}{g_{a^{\prime}}(\tau)}\right)^{2 N} \in \mathcal{F}_{N}$ if $N$ is even and $\left(\frac{g_{a}(\tau)}{g_{a^{\prime}}(\tau)}\right)^{N} \in \mathcal{F}_{N}$ if $N$ is odd. Given $a \in \mathbb{Q}^{2}$, we denote the smallest $N \in \mathbb{N}$ such that $N \cdot a \in \mathbb{Z}^{2}$ by $\operatorname{Den}(a)$.

Theorem 5.1 ([47, Ch. 2 of K1 and K2]). Assuming the notation above, the following are true:

(1) If $\gamma \in \mathrm{SL}_{2}(\mathbb{Z})$, then

$$
\left(\left.\mathfrak{t}_{a}\right|_{-1} \gamma\right)(\tau)=\mathfrak{t}_{a \gamma}(\tau)
$$

(2) If $b=\left(b_{1}, b_{2}\right) \in \mathbb{Z}^{2}$, then

$$
\mathfrak{t}_{a+b}(\tau)=e\left(1 / 2 \cdot\left(b_{1} b_{2}+b_{1}+b_{2}-b_{1} a_{2}+b_{2} a_{1}\right)\right) \mathfrak{t}_{a \gamma}(\tau) .
$$

These properties for $\mathfrak{t}_{a}$, (5.1), and the fact that $\eta(\tau)^{24}=\Delta(\tau)$ is modular on $\mathrm{SL}_{2}(\mathbb{Z})$, lead to the following properties for $g_{a}$.

Theorem 5.2 ([47, Ch. 2, Thm 1.2]). If $a \in \mathbb{Z}^{2} / N$ and $\operatorname{Den}(a)=N$, then the following are true:

(1) If $\gamma \in \mathrm{SL}_{2}(\mathbb{Z})$, then

$$
\left(\left.g_{a}^{12}\right|_{0} \gamma\right)(\tau)=g_{a \gamma}^{12}(\tau)
$$

(2) If $b=\left(b_{1}, b_{2}\right) \in \mathbb{Z}^{2}$, then

$$
g_{a+b}(\tau)=e\left(1 / 2 \cdot\left(b_{1} b_{2}+b_{1}+b_{2}-b_{1} a_{2}+b_{2} a_{1}\right)\right) g_{a}(\tau) .
$$

(3) We have that $g_{-a}(\tau)=-g_{a}(\tau)$.

(4) The $g_{a}(\tau)^{12 N}$ are modular functions on $\Gamma(N)$. Moreover, if $\gamma \in \mathrm{SL}_{2}(\mathbb{Z})$, then we have

$$
\left(\left.g_{a}^{12}\right|_{0} \gamma\right)(\tau)=g_{a \gamma}^{12}(\tau)
$$

The following theorem addresses the modularity properties of products and quotients of Siegel functions.

Theorem 5.3 ([47, Ch. 3, Lemma 5.2, Thm 5.3]). Let $N \geq 2$ be an integer, and let $\{m(a)\}_{r \in \frac{1}{N} \mathbb{Z}^{2} / \mathbb{Z}^{2}}$ be a set of integers. Then the product of Siegel functions

$$
\prod_{a \in \frac{1}{N} \mathbb{Z}^{2} / \mathbb{Z}^{2}} g_{a}^{m(a)}(\tau)
$$

belongs to $\mathcal{F}_{N}$ if $\{m(a)\}$ satisfies the following:

(1) We have that $\sum_{a} m(a)\left(N a_{1}\right)^{2} \equiv \sum_{a} m(a)\left(N a_{2}\right)^{2} \equiv 0(\bmod \operatorname{gcd}(2, N) \cdot N)$. 
(2) We have that $\sum_{a} m(a)\left(N a_{1}\right)\left(N a_{2}\right) \equiv 0(\bmod N)$.

(3) We have that $\operatorname{gcd}(12, N) \cdot \sum_{a} m(a) \equiv 0(\bmod 12)$.

Additionally, we have the following important results about the algebraicity of the singular values of the Siegel functions in relation to the singular values of the $\mathrm{SL}_{2}(\mathbb{Z})$ modular function

$$
\begin{aligned}
j(\tau) & :=\frac{\left(1+240 \sum_{n=1}^{\infty} \sum_{d \mid n} d^{3} q^{n}\right)^{3}}{q \prod_{n=1}^{\infty}\left(1-q^{n}\right)^{24}} \\
& =\frac{\eta(\tau)^{24}}{\eta(2 \tau)^{24}}+3 \cdot 2^{8}+3 \cdot 2^{16} \frac{\eta(2 \tau)^{24}}{\eta(\tau)^{24}}+2^{24} \frac{\eta(2 \tau)^{48}}{\eta(\tau)^{48}} \\
& =q^{-1}+744+196884 q+\cdots,
\end{aligned}
$$

which are well known to be algebraic by the theory of complex multiplication (for example, see [14, 26]).

Theorem 5.4 ([47, Ch. 1, Thm. 2.2]). If $\tau$ is a $C M$ point and $N=\operatorname{Den}(a)$, then the following are true:

(1) We have that $g_{a}(\tau)$ is an algebraic integer.

(2) If $N$ has at least two prime factors, then $g_{a}(\tau)$ is a unit over $\mathbb{Z}[j(\tau)]$.

(3) If $N=p^{r}$ is a prime power, then $g_{a}(\tau)$ is a unit over $\mathbb{Z}[1 / p][j(\tau)]$.

(4) If $c \in \mathbb{Z}$ and $(c, N)=1$, then $\left(g_{c a} / g_{a}\right)$ is a unit over $\mathbb{Z}[j(\tau)]$.

5.2. Galois theory of singular values of products of Siegel functions. We now recall the Galois-theoretic properties of extensions of modular function fields, and we then relate these properties to the Siegel and Klein functions.

The Galois group $\operatorname{Gal}\left(\mathcal{F}_{N} / \mathcal{F}_{1}\right)$ is isomorphic to $\mathrm{GL}_{2}(N) /\{ \pm I\}=\mathrm{GL}_{2}(\mathbb{Z} / N \mathbb{Z}) /\{ \pm I\}$ (see [47, Ch. 3, Lemma 2.1]), where $I$ is the identity matrix. This group factors naturally as

$$
\left\{\left(\begin{array}{ll}
1 & 0 \\
0 & d
\end{array}\right): d \in(\mathbb{Z} / N \mathbb{Z})^{\times}\right\} \times \mathrm{SL}_{2}(N) /\{ \pm I\},
$$

where an element $\left(\begin{array}{ll}1 & 0 \\ 0 & d\end{array}\right)$ acts on the Fourier coefficients by sending $\zeta_{N} \rightarrow \zeta_{N}^{d}$, and a matrix $\gamma \in \mathrm{SL}_{2}(\mathbb{Z})$ acts by the standard fractional linear transformation on $\tau$. If $f(\tau) \in \mathcal{F}_{N}$ and $\gamma \in \mathrm{GL}_{2}(N)$, then we use the notation $f(\tau)_{(\gamma)}:=(\gamma \circ f)(\tau)$. Applying these facts to the Siegel functions, we obtain the following.

Proposition 5.5. If $a \in \mathbb{Q}^{2}$, and $\operatorname{Den}(a)$ divides $N$, then the multiset

$$
\left\{g_{a}^{12 N}(\tau)(\gamma):=g_{a \gamma}^{12 N}(\tau): \gamma \in \mathrm{GL}_{2}(N)\right\}
$$

is a union of Galois orbits for $g_{a}^{12 N}(\tau)$ over $\mathcal{F}_{1}$.

If $\theta$ is a $C M$ point of discriminant $-D$, we define the field

$$
K_{(N)}(\theta):=\mathbb{Q}(\theta)\left(f(\theta): f \in \mathcal{F}_{N} \text { s.t. } f \text { is defined and finite at } \theta\right),
$$


and $\mathcal{H}:=\mathbb{Q}(\theta, j(\theta))$ be the Hilbert class field over $\mathbb{Q}(\theta)$. The Galois group $K_{(N)}(\theta) / \mathcal{H}$ is isomorphic to the matrix group $W_{N, \theta}$ (see [73]) defined by

$$
W_{N, \theta}=\left\{\left(\begin{array}{cc}
t-s B & -s C \\
s A & t
\end{array}\right) \in \mathrm{GL}_{2}(\mathbb{Z} / N \mathbb{Z})\right\} /\left\{ \pm\left(\begin{array}{ll}
1 & 0 \\
0 & 1
\end{array}\right)\right\},
$$

where $A x^{2}+B x+C$ is a minimal polynomial for $\theta$ over $\mathbb{Z}$. The Galois group $\operatorname{Gal}(\mathcal{H} / \mathbb{Q})$ is isomorphic to the group $\mathcal{Q}_{D}$ of primitive reduced positive-definite integer binary quadratic forms of negative discriminant $-D$. For each $Q=a x^{2}+b x y+c y^{2} \in \mathcal{Q}_{D}$, we define the corresponding $\mathrm{CM}$ point $\tau_{Q}=\frac{-b+\sqrt{-D}}{2 a}$. In order to define the action of this group, we must also define corresponding matrices $\beta_{Q} \in \mathrm{GL}_{2}(\mathbb{Z} / N \mathbb{Z})$ which we may build up by way of the Chinese Remainder Theorem and the following congruences. For each prime $p$ dividing $N$, we have the following congruences which hold $\left(\bmod p^{\operatorname{ord}_{p}(N)}\right)$ :

$$
\beta_{Q} \equiv \begin{cases}\left(\begin{array}{ll}
a & \frac{b}{2} \\
0 & 1
\end{array}\right) & \text { if } p \nmid a \\
\left(\begin{array}{cc}
-\frac{b}{2} & -c \\
1 & 0
\end{array}\right) & \text { if } p \mid a, \text { and } p \nmid c \\
\left(\begin{array}{cc}
-\frac{b}{2}-a & -\frac{b}{2}-c \\
1 & -1
\end{array}\right) & \text { if } p \mid a, \text { and } p \mid c\end{cases}
$$

if $-D \equiv 0 \quad(\bmod 4)$, and

$$
\beta_{Q} \equiv \begin{cases}\left(\begin{array}{cc}
a & \frac{b-1}{2} \\
0 & 1
\end{array}\right) & \text { if } p \nmid a \\
\left(\begin{array}{cc}
-\frac{b+1}{2} & -c \\
1 & 0
\end{array}\right) & \text { if } p \mid a, \text { and } p \nmid c \\
\left(\begin{array}{cc}
-\frac{b+1}{2}-a & \frac{1-b}{2}-c \\
1 & -1
\end{array}\right) & \text { if } p \mid a, \text { and } p \mid c\end{cases}
$$

if $-D \equiv 1(\bmod 4)$. Then given $\theta=\tau_{Q^{\prime}}$ for some $Q^{\prime} \in \mathcal{Q}_{D}$, define $\delta_{Q}(\theta):=\beta_{Q^{\prime}}^{-1} \beta_{Q}$. The Galois group $\operatorname{Gal}(\mathcal{H} / \mathbb{Q})$ can be extended into $\operatorname{Gal}\left(K_{(N)}(\theta) / \mathbb{Q}\right)$ by taking the action of a quadratic form $Q$ on the element $f(\theta) \in K_{(N)}(\theta)$ to be given by

$$
Q \circ f(\theta)=f\left(\tau_{Q}\right)_{\left(\delta_{Q}(\theta)\right)}
$$

We combine these facts into the following theorem.

Theorem 5.6. Let $F(\tau)$ be in $\mathcal{F}_{N}$ and let $\theta$ be a CM point of discriminant $-D<0$. Then the multiset

$$
\left\{F\left(\tau_{Q}\right)_{\left(\gamma \cdot \delta_{Q}(\theta)\right)}:(\gamma, Q) \in W_{\kappa, \tau} \times \mathcal{Q}_{D}\right\}
$$

is a union of the Galois orbits of $F(\theta)$ over $\mathbb{Q}$.

\section{Proofs of Theorems 1.6 and 1.7}

Here we prove Theorems 1.6 and [1.7. We shall prove these theorems using the results of the previous section. 
6.1. Reformulation of the $\Phi_{*}(m, n ; \tau)$ series. To ease the proofs of Theorems 1.6 and 1.7, we begin by reformulating each of the $\Phi_{*}(m, n ; \tau)$ series, as well as

$$
\frac{\Phi_{1 a}(m, n ; \tau)}{\Phi_{1 b}(m, n ; \tau)}
$$

as pure products of modified theta functions. These factorizations will be more useful for our purposes. In order to ease notation, for a fixed $\kappa$, if $1 \leq j<\kappa / 2$, then we let

$$
\theta_{j, \kappa}:=\theta\left(q^{j} ; q^{\kappa}\right)
$$

If $\kappa$ is even, then we let

$$
\theta_{\kappa / 2, \kappa}:=\left(q^{\kappa / 2} ; q^{\kappa}\right)_{\infty}=\theta\left(q^{\kappa / 2} ; q^{2 \kappa}\right),
$$

which is a square root of $\theta\left(q^{\kappa / 2} ; q^{\kappa}\right)$.

The reformulations below follow directly from (1.15) by making use of the fact that

$$
\frac{\left(q^{\kappa} ; q^{\kappa}\right)_{\infty}}{(q)_{\infty}}=\prod_{j=1}^{\lfloor\kappa / 2\rfloor} \theta_{j, \kappa}
$$

Lemma 6.1. Let $m$ and $n$ be positive integers and $\kappa_{*}=\kappa_{*}(m, n)$ as in (1.14). Then the following are true:

(1a) With $\kappa=\kappa_{1}$,

$$
\Phi_{1 a}(m, n ; \tau)=q^{\frac{m n(4 m n-4 m+2 n-3)}{12 \kappa}} \prod_{j=1}^{m} \theta_{j, \kappa}^{-1} \prod_{j=1}^{m+n} \theta_{j, \kappa}^{-\min (m, n-1,\lceil j / 2\rceil-1)} .
$$

(1b) With $\kappa=\kappa_{1}$,

$$
\Phi_{1 b}(m, n ; \tau)=q^{\frac{m n(4 m n+2 m+2 n+3)}{12 \kappa}} \prod_{j=1}^{m+n} \theta_{j, \kappa}^{-\min (m, n,\lfloor j / 2\rfloor)}
$$

(2) With $\kappa=\kappa_{2}$,

$$
\Phi_{2}(m, n ; \tau)=q^{\frac{m(2 n+1)(2 m n-m+n-1)}{12 \kappa}} \prod_{j=1}^{m} \theta_{j, \kappa}^{-1} \prod_{j=1}^{m+n+1} \theta_{j, \kappa}^{-\min (m, n-1,\lceil j / 2\rceil-1)} \prod_{j=n}^{\lfloor(m+n) / 2\rfloor} \theta_{2 j+1, \kappa}^{-1} .
$$

(3) For $n \geq 2$ and $\kappa=\kappa_{3}$,

$$
\Phi_{3}(m, n ; \tau)=q^{\frac{m(2 n-1)(2 m n+n+1)}{12 \kappa}} \prod_{j=1}^{m} \theta\left(q^{2 j} ; q^{\kappa}\right)^{-1} \prod_{j=1}^{m+n} \theta_{j, \kappa}^{-\min (m, n-2,\lceil j / 2\rceil-1)} \prod_{j=n}^{\lfloor(m+n+1) / 2\rfloor} \theta_{2 j-1, \kappa}^{-1} .
$$

Moreover, with $\kappa=\kappa_{1}(m, n)$,

$$
\Psi_{1}(m, n ; \tau):=\frac{\Phi_{1 a}(m, n ; \tau)}{\Phi_{1 b}(m, n ; \tau)}=q^{-\frac{m n(m+1)}{2 \kappa}} \prod_{j=1}^{m} \frac{\theta\left(q^{2 j} ; q^{\kappa}\right)}{\theta\left(q^{j} ; q^{\kappa}\right)}
$$


and with $\kappa=\kappa_{2}(m, n)=\kappa_{3}(m, n+1)$,

$$
\Psi_{2}(m, n ; \tau):=\frac{\Phi_{2}(m, n ; \tau)}{\Phi_{3}(m, n+1 ; \tau)}=q^{-\frac{m(m+1)(2 n+1)}{4 \kappa}} \prod_{j=1}^{m} \frac{\theta\left(q^{2 j} ; q^{\kappa}\right)}{\theta\left(q^{j} ; q^{\kappa}\right)} .
$$

Proof. Since the proofs of the four cases are essentially the same, we only prove Lemma 6.1 (1a).

Let $\varphi=m n(4 m n-4 m+2 n-3) /\left(12 \kappa_{1}\right)$. By Theorem 1.1, we have that

$$
\begin{aligned}
\Phi_{1 a}(m, n ; \tau) & =q^{\varphi} \cdot \frac{\left(q^{\kappa} ; q^{\kappa}\right)_{\infty}^{n}}{(q)_{\infty}^{n}} \prod_{i=1}^{n} \theta\left(q^{i+m} ; q^{\kappa}\right) \prod_{1 \leq i<j \leq n} \theta\left(q^{j-i}, q^{i+j-1} ; q^{\kappa}\right) \\
& =q^{\varphi} \cdot \frac{\left(q^{\kappa} ; q^{\kappa}\right)_{\infty}^{m}}{(q)_{\infty}^{m}} \prod_{i=1}^{m} \theta\left(q^{i+1} ; q^{\kappa}\right) \prod_{1 \leq i<j \leq m} \theta\left(q^{j-i}, q^{i+j+1} ; q^{\kappa}\right) .
\end{aligned}
$$

Using the simple identity

$$
\frac{\left(q^{\kappa} ; q^{\kappa}\right)_{\infty}}{(q)_{\infty}}=\prod_{j=1}^{m+n} \theta\left(q^{j} ; q^{\kappa}\right)^{-1}
$$

we can rewrite these two forms as

$$
\begin{aligned}
\Phi_{1 a}(m, n ; \tau) & =q^{\varphi} \cdot \frac{\prod_{i=1}^{n} \theta\left(q^{i+m} ; q^{\kappa}\right)}{\prod_{i=1}^{m+n} \theta\left(q^{j} ; q^{\kappa}\right)} \cdot \prod_{j=2}^{n} \frac{\prod_{i=1}^{j-1} \theta\left(q^{j-i}, q^{i+j-1} ; q^{\kappa}\right)}{\prod_{i=1}^{m+n} \theta\left(q^{i}, q^{\kappa}\right)} \\
& =q^{\varphi} \cdot \frac{\prod_{i=1}^{m} \theta\left(q^{i+1} ; q^{\kappa}\right)}{\prod_{i=1}^{m+n} \theta\left(q^{j} ; q^{\kappa}\right)} \cdot \prod_{j=2}^{m} \frac{\prod_{i=1}^{j-1} \theta\left(q^{j-i}, q^{i+j+1} ; q^{\kappa}\right)}{\prod_{i=1}^{m+n} \theta\left(q^{i} ; q^{\kappa}\right)} .
\end{aligned}
$$

If $m \geq n-1$ then the first identity reduces to

$$
\begin{aligned}
\Phi_{1 a}(m, n ; \tau) & =q^{\varphi} \cdot\left(\prod_{j=1}^{m} \theta\left(q^{j} ; q^{\kappa}\right)\right)^{-1} \prod_{j=2}^{n}\left(\prod_{i=2 j-1}^{m+n} \theta\left(q^{i} ; q^{\kappa}\right)\right)^{-1} \\
& =q^{\varphi} \cdot\left(\prod_{j=1}^{m} \theta\left(q^{j} ; q^{\kappa}\right)\right)^{-1} \prod_{j=1}^{n-1}\left(\prod_{i=2 j+1}^{m+n} \theta\left(q^{i} ; q^{\kappa}\right)\right)^{-1} .
\end{aligned}
$$

If $m \leq n-1$ then the second identity reduces to

$$
\begin{aligned}
\Phi_{1 a}(m, n ; \tau)=q^{\varphi} \cdot\left(\theta\left(q ; q^{\kappa}\right) \prod_{j=m+2}^{m+n} \theta\left(q^{j} ; q^{\kappa}\right)\right)^{-1} \\
\times \prod_{j=2}^{m}\left(\theta\left(q^{j}, q^{j+1} ; q^{\kappa}\right) \prod_{i=2 j+1}^{m+n} \theta\left(q^{i} ; q^{\kappa}\right)\right)^{-1} \\
=q^{\varphi} \cdot\left(\prod_{j=1}^{m} \theta\left(q^{j} ; q^{\kappa}\right)\right)^{-1} \prod_{j=1}^{m}\left(\prod_{i=2 j+1}^{m+n} \theta\left(q^{i} ; q^{\kappa}\right)\right)^{-1} .
\end{aligned}
$$

Together these imply Lemma 6.1 (1a). 
Since the modified $\theta$-functions $\theta\left(q^{\ell} ; q^{\kappa}\right)$ are essentially Siegel functions (up to powers of $q$ ), we can immediately rewrite Lemma 6.1 in terms of modular functions. We shall omit the proofs for brevity.

Lemma 6.2. Let $m$ and $n$ be positive integers and $\kappa_{*}=\kappa_{*}(m, n)$ as in (1.14). Then the following are true:

(1a) With $\kappa=\kappa_{1}$,

$$
\Phi_{1 a}(m, n ; \tau)=\prod_{j=1}^{m} g_{j / \kappa, 0}(\kappa \tau)^{-1} \prod_{j=1}^{m+n} g_{j / \kappa, 0}(\kappa \tau)^{-\min (m, n-1,\lceil j / 2\rceil-1)} .
$$

(1b) With $\kappa=\kappa_{1}$,

$$
\Phi_{1 b}(m, n ; \tau)=\prod_{j=1}^{m+n} g_{j / \kappa, 0}(\kappa \tau)^{-\min (m, n,\lfloor j / 2\rfloor}
$$

(2) With $\kappa=\kappa_{2}$,

$$
\begin{aligned}
\Phi_{2}(m, n ; \tau)=g_{\frac{1}{4}, 0}(2 \kappa \tau)^{-\min (m, n-1)-\delta} \prod_{j=1}^{m} g_{\frac{j}{\kappa}, 0}(\kappa \tau)^{-1} & \\
& \times \prod_{j=1}^{m+n} g_{\frac{j}{\kappa}, 0}(\kappa \tau)^{-\min (m, n-1,\lceil j / 2\rceil-1)} \prod_{j=n}^{\lfloor(m+n-1) / 2\rfloor} g_{\frac{(2 j+1)}{\kappa}, 0}(\kappa \tau)^{-1} .
\end{aligned}
$$

(3) For $n \geq 2$ and $\kappa=\kappa_{3}$,

$$
\begin{aligned}
\Phi_{3}(m, n ; \tau)=g_{\frac{1}{4}, 0}(2 \kappa \tau)^{-\min (m, n-2)-\delta} & \prod_{j=1}^{m} g_{\frac{2 j}{\kappa}, 0}(\kappa \tau)^{-1} \\
& \times \prod_{j=1}^{m+n-1} g_{\frac{j}{\kappa}, 0}(\kappa \tau)^{-\min (m, n-2,\lceil j / 2\rceil-1)} \prod_{j=n}^{\lfloor(m+n) / 2\rfloor} g_{\frac{(2 j-1)}{\kappa}, 0}(\kappa \tau)^{-1} .
\end{aligned}
$$

(4) With $\kappa=\kappa_{1}$,

$$
\Psi_{1}(m, n ; \tau):=\frac{\Phi_{1 a}(m, n ; \tau)}{\Phi_{1 b}(m, n ; \tau)}=\prod_{j=1}^{m} \frac{g_{\frac{2 j}{\kappa}, 0}(\kappa \tau)}{g_{\frac{j}{\kappa}, 0}(\kappa \tau)} .
$$

(5) With $\kappa=\kappa_{2}(m, n)=\kappa_{3}(m, n+1)$,

$$
\Psi_{2}(m, n ; \tau):=\frac{\Phi_{2}(m, n ; \tau)}{\Phi_{3}(m, n+1 ; \tau)}=\prod_{j=1}^{m} \frac{g_{\frac{2 j}{\kappa}, 0}(\kappa \tau)}{g_{\frac{j}{\kappa}, 0}(\kappa \tau)} .
$$

In parts (2) and (3) we have $\delta=0$ or 1 depending on if $\kappa / 2$ is even or odd respectively. 
6.2. Proofs of Theorems $\mathbf{1 . 6}$ and 1.7. We now apply the results in Section 5 to prove Theorems 1.6 and 1.7.

Proof of Theorem 1.6 (1) and (2). Lemma 6.2 shows that each of the $\Phi_{*}(m, n ; \tau)$ is exactly a pure product of Siegel functions. Therefore, we may apply Theorem 5.2 directly to each of the Siegel function factors, and as a consequence to each $\Phi_{*}(m, n ; \tau)$.

Since by Theorem 5.2 (4), $g_{a}(\tau)^{12 N}$ is in $\mathcal{F}_{N}$ if $N=\operatorname{Den}(a)$, we may take $N=\kappa_{*}(m, n)$, and so we have that $\Phi_{*}(m, n ; \tau)^{12 \kappa} \in \mathcal{F}_{\kappa_{*}(m, n)}$. We now apply Theorem 5.6 to obtain Theorem 1.6 (1) and (2).

Sketch of the Proof of Theorem 1.6 (3). By Theorem [1.6 (2), we have that this multiset consists of multiple copies of a single Galois orbit of conjugates over $\mathbb{Q}$. Therefore to complete the proof, it suffices to show that the given conditions imply that there are singular values which are not repeated. To this end, we focus on those CM points with maximal imaginary parts. Indeed, because each $\Phi_{*}(m, n ; \tau)$ begins with a negative power of $q$, one generically expects that these corresponding singular values will be the ones with maximal complex absolute value.

To make this argument precise requires some cumbersome but unenlightening details (which we omit)]. One begins by observing why the given conditions are necessary. For small $\kappa$ it can happen that the matrices in $W_{\kappa, \tau}$ permute the Siegel functions in the factorizations of $\Phi_{*}(m, n ; \tau)$ obtained in Lemma 6.2. However, if $\kappa>9$, then this does not happen. The condition that $\operatorname{gcd}\left(D_{0}, \kappa\right)=1$ is required for a similar reason. More precisely, the group does not act faithfully. However, under these conditions, the only obstruction to the conclusion would be a nontrivial identity between the evaluations of two different modular functions. In particular, under the given assumptions, we may view these functions as a product of distinct Siegel functions. Therefore, the proof follows by studying the asymptotic properties of the CM values of individual Siegel functions, and then considering the $\Phi_{*}$ functions as a product of these values.

The relevant asymptotics arise by considering, for each $-D$, a canonical CM point with discriminant $-D$. Namely, we let

$$
\tau_{*}:=\left\{\begin{array}{lll}
\frac{\sqrt{-D}}{2} & \text { if }-D \equiv 0 \quad(\bmod 4), \\
\frac{1+\sqrt{-D}}{2} & \text { if }-D \equiv 1 \quad(\bmod 4) .
\end{array}\right.
$$

By the theory of reduced binary quadratic forms, these points are the CM points with maximal imaginary parts corresponding to reduced forms with discriminant $-D$. Moreover, every other CM point with discriminant $-D$ has imaginary part less than $|\sqrt{-D}| / 3$. Now the singular values of each Siegel function then essentially arise from the values of the second Bernoulli polynomial. The point is that one can uniformly estimate the infinite product portion of each singular value, and it turns out that they are exponentially close to the number 1. By assembling these estimates carefully, one obtains the result.

\footnotetext{
${ }^{7}$ A similar analysis is carried out in detail by Jung, Koo, and Shin in [41, Sec. 4].
} 
Proof of Theorem 1.7. Lemma 6.2 reformulates each $\Phi_{*}$ function in terms of products of negative powers of Siegel functions of the form $g_{j / \kappa, 0}(\kappa \tau)$, where $1 \leq j \leq \kappa / 2$, and $g_{1 / 4,0}(2 \kappa \tau)$, when $\kappa$ is even. Theorem $5.4(1)$ then implies Theorem $1.7(1)$.

Since $\operatorname{Den}(j / \kappa, 0)$ may be any divisor of $\kappa$, and since $j(\tau)$ is an algebraic integer [14, 26], Theorem 5.4 (2) and (3) imply Theorem 1.7 (2).

Using Theorem 6.2 (5), we have that

$$
\frac{\Phi_{1 a}(m, n ; \tau)}{\Phi_{1 b}(m, n ; \tau)}=\prod_{j=1}^{m} \frac{g_{\frac{2 j}{\kappa}, 0}(\kappa \tau)}{g_{\frac{j}{\kappa}, 0}(\kappa \tau)},
$$

where $\kappa=\kappa_{1}=2 m+2 n+1$. Since $\kappa$ is odd, Theorem 5.4 (4) implies that each term

$$
\frac{g_{\frac{2 j}{\kappa}, 0}(\kappa \tau)}{g_{\frac{j}{\kappa}, 0}(\kappa \tau)}
$$

in the product is a unit. Therefore, Theorem 1.7 (3) follows.

\section{EXAMPLES}

Here we give two examples of the main results in this paper.

Example. This is a detailed discussion of the example in Section 1.

Consider the $q$-series

$$
\begin{aligned}
\Phi_{1 a}(2,2 ; \tau) & =q^{1 / 3} \prod_{n=1}^{\infty} \frac{\left(1-q^{9 n}\right)}{\left(1-q^{n}\right)} \\
& =q^{1 / 3}+q^{4 / 3}+2 q^{7 / 3}+3 q^{10 / 3}+5 q^{13 / 3}+7 q^{16 / 3}+\cdots,
\end{aligned}
$$

and

$$
\begin{aligned}
\Phi_{1 b}(2,2 ; \tau) & =q \prod_{n=1}^{\infty} \frac{\left(1-q^{9 n}\right)\left(1-q^{9 n-1}\right)\left(1-q^{9 n-8}\right)}{\left(1-q^{n}\right)\left(1-q^{9 n-4}\right)\left(1-q^{9 n-5}\right)} \\
& =q+q^{3}+q^{4}+3 q^{5}+3 q^{6}+5 q^{7}+6 q^{8}+\cdots
\end{aligned}
$$

For $\tau=\mathrm{i} / 3$, the first 100 coefficients of the $q$-series respectively give the numerical approximations

$$
\begin{aligned}
& \Phi_{1 a}(2,2 ; \mathrm{i} / 3)=0.577350 \ldots \stackrel{?}{=} \frac{1}{\sqrt{3}} \\
& \Phi_{1 b}(2,2 ; \mathrm{i} / 3)=0.125340 \ldots
\end{aligned}
$$

Here we have that $\kappa_{1}(2,2)=9$. Theorem 5.3 tells us that $\Phi_{1 a}(2,2 ; \tau)^{3}$ and $\Phi_{1 b}(2,2 ; \tau)^{3}$ are in $\mathcal{F}_{9}$, so we may use Theorem 5.6 to find the conjugates of the values of the functions at $\tau=\mathrm{i} / 3$. We have $\kappa_{1}(2,2) \cdot \mathrm{i} / 3=3 \mathrm{i}$ and

$$
W_{9,3 i}=\left\{\left(\begin{array}{ll}
t & 0 \\
s & t
\end{array}\right) \in \mathrm{GL}_{2}(\mathbb{Z} / 9 \mathbb{Z})\right\} /\left\{ \pm\left(\begin{array}{ll}
1 & 0 \\
0 & 1
\end{array}\right)\right\}
$$


which has 27 elements. However each of these acts like the identity on $\Phi_{1 a}(2,2 ; \tau)$, and the group has an orbit of size three when acting on $\Phi_{1 b}(2,2 ; \tau)$. The set $\mathcal{Q}_{36}$ has two elements $Q_{1}=x^{2}+9 y^{2}$ and $Q_{2}=2 x^{2}+2 x y+5 y^{2}$. These qive us $\beta_{Q_{1}}$ which is the identity, and $\beta_{Q_{2}}=\left(\begin{array}{ll}2 & 1 \\ 0 & 1\end{array}\right)$. Therefore $\Phi_{1 a}(2,2 ; \mathrm{i} / 3)^{3}$ has only one other conjugate,

$$
\left(g_{2 / 9,1 / 9}\left(\frac{-1+3 \mathrm{i}}{2}\right) g_{4 / 9,2 / 9}\left(\frac{-1+3 \mathrm{i}}{2}\right) g_{6 / 9,3 / 9}\left(\frac{-1+3 \mathrm{i}}{2}\right) g_{8 / 9,4 / 9}\left(\frac{-1+3 \mathrm{i}}{2}\right)\right)^{-3}
$$

although the multiset described in Theorem 1.6 (2) contains 27 copies of these two numbers. On the other hand, $\Phi_{1 b}(2,2 ; \mathrm{i} / 3)^{3}$ has an orbit of six conjugates, and the multiset from Theorem [1.6 (2) contains nine copies of this orbit. Theorem 1.7 (2) tells us that $\Phi_{1 a}(2,2 ; \mathrm{i} / 3)$ and $\Phi_{1 b}(2,2 ; \mathrm{i} / 3)$ may have denominators which are powers of three, whereas Theorem 1.7 (1) tells us that their inverses are algebraic integers. Therefore, we find the minimal polynomials for the inverses and then invert the polynomials. In this way, we find that $\Phi_{1 a}(2,2 ; \mathrm{i} / 3)$ and $\Phi_{1 b}(2,2 ; \mathrm{i} / 3)$ are roots of the irreducible polynomials

$$
\begin{gathered}
3 x^{2}-1 \\
19683 x^{18}-80919 x^{12}+39366 x^{9}+11016 x^{6}+486 x^{3}-1 .
\end{gathered}
$$

The full polynomials whose roots are the elements of the multisets corresponding to $\Phi_{1 a}(2,2 ; \mathrm{i} / 3)^{3}$ and $\Phi_{1 b}(2,2 ; \mathrm{i} / 3)^{3}$, counting multiplicity are

$$
\begin{gathered}
\left(27 x^{2}-1\right)^{27} \\
\left(19683 x^{6}-80919 x^{4}-39366 x^{3}+11016 x^{2}-486 x^{2}-1\right)^{9} .
\end{gathered}
$$

Applying Theorem $1.7(2)$, we find that $\sqrt{3} \Phi_{1 a}(2,2 ; \mathrm{i} / 3)$ and $\sqrt{3} \Phi_{1 b}(2,2 ; \mathrm{i} / 3)$ are units and roots of the polynomials

$$
\begin{gathered}
x-1 \\
x^{18}+6 x^{15}-93 x^{12}-304 x^{9}+420 x^{6}-102 x^{3}+1 .
\end{gathered}
$$

Lastly, Theorem 1.7 (3) applies, and we know that the ratio

$$
\begin{aligned}
\frac{\Phi_{1 a}(2,2 ; \tau)}{\Phi_{1 b}(2,2 ; \tau)} & =q^{-2 / 3} \prod_{n=1}^{\infty} \frac{\left(1-q^{9 n-4}\right)\left(1-q^{9 n-5}\right)}{\left(1-q^{9 n-1}\right)\left(1-q^{9 n-8}\right)} \\
& =q^{-2 / 3}\left(1+q+q^{2}+q^{3}-q^{5}-q^{6}-q^{7}+\cdots\right)
\end{aligned}
$$

evaluates to a unit at $\tau=\mathrm{i} / 3$. In fact we find that

$$
\frac{\Phi_{1 a}(2,2 ; \mathrm{i} / 3)}{\Phi_{1 b}(2,2 ; \mathrm{i} / 3)}=4.60627 \ldots
$$

is a unit. Indeed, it is a root of

$$
x^{18}-102 x^{15}+420 x^{12}-304 x^{9}-93 x^{6}+6 x^{3}+1 .
$$


Example. Here we give an example which illustrates the second remark after Theorem 1.7. This is the discussion concerning ratios of singular values of $\Phi_{2}$ and $\Phi_{3}$ with the same $\kappa_{*}$. Here we show that these ratios are not generically algebraic integral units as Theorem $1.7(3)$ guarantees for the $\mathrm{A}_{2 n}^{(2)}$ cases.

We consider $\Phi_{2}(1,1 ; \tau)$ and $\Phi_{3}(1,2 ; \tau)$, with $\tau=\sqrt{-1 / 3}$. For these example we have $\kappa_{2}(1,1)=\kappa_{3}(1,2)=6$. A short computation by way of the $q$-series shows that

$$
\Phi_{2}(1,1 ; \sqrt{-1 / 3})=0.883210 \ldots
$$

and

$$
\Phi_{3}(1,2 ; \sqrt{-1 / 3})=0.347384 \ldots
$$

Since $\Phi_{2}(1,1 ; \tau)^{24}$ and $\Phi_{3}(1,2 ; \tau)^{24}$ are in $\mathcal{F}_{12}$, we find that

$$
\Phi_{2}(1,1 ; \sqrt{-1 / 3})^{24} \text { and } \Phi_{3}(1,2 ; \sqrt{-1 / 3})^{24}
$$

each have one other conjugate, namely

$$
\left(g_{1 / 2,1 / 3}(\sqrt{-4 / 3}) \cdot g_{1 / 4,0}(2 \sqrt{-4 / 3})\right)^{-24} \text { and }\left(g_{0,1 / 3}(\sqrt{-4 / 3}) \cdot g_{1 / 2,0}(2 \sqrt{-4 / 3})\right)^{-24}
$$

respectively, and the corresponding multisets described in Theorem 1.6 (2) each contain six copies of the respective orbits. In this way we find that $\Phi_{2}(1,1 ; \sqrt{-1 / 3})$ is a root of

$$
2^{20} x^{48}-2^{12} \cdot 13 x^{24}+1
$$

and $\Phi_{3}(1,2 ; \sqrt{-1 / 3})$ is a root of

$$
2^{20} 3^{12} x^{48}-12^{6} \cdot 35113 x^{24}+1 .
$$

Therefore, their ratio

$$
\frac{\Phi_{2}(1,1 ; \sqrt{-1 / 3})}{\Phi_{3}(1,2 ; \sqrt{-1 / 3})}=2.542459 \ldots
$$

is not a unit. Its minimal polynomial is

$$
x^{4}-6 x^{2}-3
$$

\section{REFERENCES}

1. G. E. Andrews, An analytic generalization of the Rogers-Ramanujan identities for odd moduli, Proc. Nat. Acad. Sci. USA 71 (1974), 4082-4085.

2. G. E. Andrews, Problems and prospects for basic hypergeometric functions, in Theory and Application of Special Functions, pp. 191-224, Math. Res. Center, Univ. Wisconsin, 35, Academic Press, New York, 1975.

3. G. E. Andrews, The Theory of Partitions, Encyclopedia of Mathematics and its Applications, Vol. 2, Addison-Wesley, Reading, Massachusetts, 1976.

4. G. E. Andrews, R. J. Baxter and P. J. Forrester, Eight-vertex SOS model and generalized RogersRamanujan-type identities, J. Statist. Phys. 35 (1984), 193-266.

5. G. E. Andrews, A. Schilling and S. O. Warnaar, An $A_{2}$ Bailey lemma and Rogers-Ramanujan-type identities, J. Amer. Math. Soc. 12 (1999), 677-702.

6. C. Armond and O. T. Dasbach, Rogers-Ramanujan identities and the head and tail of the colored Jones polynomial, arXiv:1106.3948. 
7. R. Askey and M. E. H. Ismail, A generalization of ultraspherical polynomials, in Studies in pure mathematics, pp. 55-78, Birkhäuser, Basel, 1983.

8. N. Bartlett and S. O. Warnaar, Hall-Littlewood polynomials and characters of affine Lie algebras, Adv. in Math. 285 (2015), 1066-1105.

9. R. J. Baxter, Rogers-Ramanujan identities in the hard hexagon model, J. Statist. Phys. 26 (1981), 427-452.

10. A. Berkovich and B. M. McCoy, Rogers-Ramanujan identities: a century of progress from mathematics to physics, Proceedings of the International Congress of Mathematicians, Vol. III (Berlin, 1998), Doc. Math. 1998, Extra Vol. III, 163-172.

11. B. C. Berndt, H. H. Chan and L.-C. Zhang, Explicit evaluations of the Rogers-Ramanujan continued fraction, J. Reine Angew. Math. 480 (1996), 141-159.

12. B. C. Berndt and R. A. Rankin, Ramanujan. Letters and commentary, Amer. Math. Soc., Providence, RI, 1995.

13. D. M. Bressoud, An analytic generalization of the Rogers-Ramanujan identities with interpretation, Quart. J. Maths. Oxford (2) 31 (1980), 385-399.

14. A. Borel, S. Chowla, C. S. Herz, K. Iwasawa and J.-P. Serre, Seminar on Complex Multiplication, Lecture Notes in Mathematics, No. 21, Springer-Verlag, Berlin-New York 1966.

15. D. M. Bressoud, On partitions, orthogonal polynomials and the expansion of certain infinite products, Proc. London Math. Soc. (3) 42 (1981), 478-500.

16. K. Bringmann, C. Calinescu, A. Folsom, and S. Kimport, Graded dimensions of principal subspaces and modular Andrews-Gordon series, Commun. Contemp. Math. 16 (2014), 1350050, 20 pp.

17. K. Bringmann, A. Folsom, and K. Mahlburg, Quasimodular forms and $\operatorname{sl}(m \mid m)^{\wedge}$ characters, Ramanujan J. 36 (2015), 103-116.

18. K. Bringmann and A. Folsom, On the asymptotic behavior of Kac-Wakimoto characters, Proc. Amer. Math. Soc. 141 (2013), 1567-1576.

19. K. Bringmann and A. Folsom, Almost harmonic Maass forms and Kac-Wakimoto characters, J. Reine Angew. Math. 694 (2014), 179-202.

20. K. Bringmann and K. Mahlburg, Asymptotic formulas for coefficients of Kac-Wakimoto characters, Math. Proc. Cambridge Phil. Soc. 155 (2013), 51-72.

21. K. Bringmann and K. Ono, Some characters of Kac and Wakimoto and nonholomorphic modular functions, Math. Ann. 345 (2009), 547-558.

22. K. Bringmann, K. Ono and R. Rhoades, Eulerian series as modular forms, J. Amer. Math. Soc. 21 (2008), 1085-1104.

23. C. Bruschek, H. Mourtada and J. Schepers, Arc spaces and the Rogers-Ramanujan identities, Ramanujan J. 30 (2013), 9-38.

24. B. Cais and B. Conrad, Modular curves and Ramanujan's continued fraction, J. Reine Angew. Math. 597 (2006), 27-104.

25. I. Cherednik and B. Feigin, Rogers-Ramanujan type identities and Nil-DAHA, Adv. in Math. 248 (2013), 1050-1088.

26. D. A. Cox, Primes of the Form $x^{2}+n y^{2}$. Fermat, Class Field Theory, and Complex Multiplication, J. Wiley and Sons, Inc., New York, 1989.

27. W. Duke, Continued fractions and modular functions, Bull. Amer. Math. Soc. 42 (2005), 137-162.

28. J. L. Dupont and C.-H. Sah, Dilogarithm identities in conformal field theory and group homology, Comm. Math. Phys. 161 (1994), 265-282.

29. F. Dyson, A walk through Ramanujan's garden, Ramanujan revisited, AMS, Providence, 2001, 7-28.

30. B. Feigin, O. Foda and T. Welsh, Andrews-Gordon type identities from combinations of Virasoro characters, Ramanujan J. 17 (2008), 33-52. 
31. B. Feigin and E. Frenkel, Coinvariants of nilpotent subalgebras of the Virasoro algebra and partition identities, in I. M. Gel'fand Seminar, pp. 139-148, Adv. Soviet Math., 16, Part 1, AMS, Providence, RI, 1993.

32. J. Fulman, The Rogers-Ramanujan identities, the finite general linear groups, and the Hall-Littlewood polynomials, Proc. Amer. Math. Soc. 128 (2000), 17-25.

33. J. Fulman, A probabilistic proof of the Rogers-Ramanujan identities, Bull. London Math. Soc. 33 (2001), 397-407.

34. I. Frenkel, J. Lepowsky and A. Meurman, Vertex operator algebras and the Monster, Pure and Applied Mathematics, No. 134, Academic Press, Inc., Boston, MA, 1988.

35. K. Garrett, M. E. H. Ismail and D. Stanton, Variants of the Rogers-Ramanujan identities, Adv. in Appl. Math. 23 (1999), 274-299.

36. B. Gordon, A combinatorial generalization of the Rogers-Ramanujan identities, Amer. J. Math. 83 (1961), 393-399.

37. E. Gorsky, A. Oblomkov and J. Rasmussen, On stable Khovanov homology of torus knots, Exp. Math. 22 (2013), 265-281

38. R. A. Gustafson, The Macdonald identities for affine root systems of classical type and hypergeometric series very-well-poised on semisimple Lie algebras, in Ramanujan International Symposium on Analysis, pp. 187-224, Macmillan of India, New Delhi, 1989.

39. K. Hikami, Volume conjecture and asymptotic expansion of q-series, Experiment. Math. 12 (2003), 319-337.

40. K. Hikami, q-Series and L-functions related to half-derivatives of the Andrews-Gordon identity, Ramanujan J. 11 (2006), 175-197.

41. H. Y. Jung, J. K. Koo and D. H. Shin, On some arithmetic properties of Siegel functions (II), Forum Math. 26, (2014), 25-58.

42. V. G. Kac, Infinite-dimensional algebras, Dedekind's $\eta$-function, classical Möbius function and the very strange formula, Adv. in Math. 30 (1978), 85-136.

43. V. G. Kac, Infinite-dimensional Lie Algebras, 3rd Edition, Cambridge University Press, Cambridge, 1990.

44. R. Kedem, B. M. McCoy and E. Melzer, The sums of Rogers, Schur and Ramanujan and the BoseFermi correspondence in $(1+1)$-dimensional quantum field theory, in Recent Progress in Statistical Mechanics and Quantum Field Theory, pp. 195-219, World Sci. Publ., River Edge, NJ, 1995.

45. A. N. Kirillov, New combinatorial formula for modified Hall-Littlewood polynomials, in q-Series from a Contemporary Perspective, pp. 283-333, Contemp. Math., 254, AMS, Providence, RI, 2000.

46. C. Krattenthaler, Advanced determinant calculus, Sém. Lothar. Combin. 42 (1999), Art. B42q, 41pp.

47. D. Kubert and S. Lang, Modular Units, Grundlehren der Mathematischen Wissenchaften 244, Springer-Verlag, New York-Berlin, 1981.

48. J. Lepowsky, Generalized Verma modules, loop space cohomology and Macdonald-type identities, Ann. Sci. Ecole Norm. Sup. 12 (1979), 169-234.

49. J. Lepowsky, Affine Lie algebras and combinatorial identities in Lie algebras and related topics, pp. 130-156, Lecture Notes in Mathematics No. 933, Springer, Berlin-New York, 1982.

50. J. Lepowsky and S. Milne, Lie algebras and classical partition identities, Proc. Nat. Acad. Sci. U.S.A. 75 (1978), 578-579.

51. J. Lepowsky and S. Milne, Lie algebraic approaches to classical partition identities, Adv. in Math. 29 (1978), 15-59.

52. J. Lepowsky and M. Primc, Structure of the standard modules for the affine Lie algebra $A_{1}^{(1)}$, Contemporary Mathematics, No. 46, AMS, Providence, RI, 1985.

53. J. Lepowsky and R. L. Wilson, The Rogers-Ramanujan identities: Lie theoretic interpretation and proof, Proc. Nat. Acad. Sci. U.S.A. 78 (1981), 699-701. 
54. J. Lepowsky and R. L. Wilson, A new family of algebras underlying the Rogers-Ramanujan identities and generalizations, Proc. Nat. Acad. Sci. U.S.A. 78 (1981), 7254-7258.

55. J. Lepowsky and R. L. Wilson, A Lie theoretic interpretation and proof of the Rogers-Ramanujan identities, Adv. in Math. 45 (1982), 21-72.

56. J. Lepowsky and R. L. Wilson, The structure of standard modules. I. Universal algebras and the Rogers-Ramanujan identities, Inv. Math. 77 (1984), 199-290.

57. I. G. Macdonald, Affine root systems and Dedekind's $\eta$-function, Invent. Math. 15 (1972), 91-143.

58. I. G. Macdonald, Symmetric functions and Hall polynomials, 2nd edition, Oxford Univ. Press, New York, 1995.

59. P. A. MacMahon, Combinatory Analysis, Vol. I and II, Chelsea Publishing Co., New York 1960.

60. H. McKean and V. Moll, Elliptic Curves: Function Theory, Geometry, Arithmetic, Cambridge Univ. Press, Cambridge, 1997.

61. S. C. Milne, The $C_{l}$ Rogers-Selberg identity, SIAM J. Math. Anal. 25 (1994), 571-595.

62. A. Meurman and M. Primc, Vertex operator algebras and representations of affine Lie algebras, Acta Appl. Math. 44 (1996), 207-215.

63. K. Ono, Unearthing the visions of a master: harmonic Maass forms and number theory, in HarvardMIT Current Developments in Mathematics, 2008, pp. 347-454, Int. Press, Somerville, MA, 2009.

64. E. M. Rains, $B C_{n}$-symmetric polynomials, Transform. Groups 10 (2005), 63-132.

65. E. M. Rains and M. Vazirani, Vanishing integrals of Macdonald and Koornwinder polynomials, Transform. Groups 12 (2007), 725-759.

66. E. M. Rains and S. O. Warnaar, Bounded Littlewood identities, arXiv:1506.02755.

67. K. G. Ramanathan, On Ramanujan's continued fraction, Acta Arith, 43 (1984), 209-226.

68. B. Richmond and G. Szekeres, Some formulas related to dilogarithms, the zeta function and the Andrews-Gordon identities, J. Austral. Math. Soc. Ser. A 31 (1981), 362-373.

69. L. J. Rogers, Second memoir on the expansion of certain infinite products, Proc. London Math. Soc. 25 (1894), 318-343.

70. L. J. Rogers, Proof of cerytain identities in combinatory analysis, Proc. Camb. Phil. Soc. 19 (1919), $214-216$.

71. I. Schur, Ein Beitrag zur Additiven Zahlen theorie und zur Theorie der Kettenbrüche, S.-B. Preuss. Akad. Wiss. Phys. Math. Klasse (1917), 302-321.

72. A. Selberg, Über einige arithmetische Identitäten, Avh. Norske Vid.-Akad. Oslo. I. Mat.-Naturv. Kl. 8 (1936), 1-23.

73. G. Shimura, Introduction to the Arithmetic Theory of Automorphic Functions, Princeton University Press, Princeton, NJ, 1994.

74. J. R. Stembridge, Hall-Littlewood functions, plane partitions, and the Rogers-Ramanujan identities, Trans. Amer. Math. Soc. 319 (1990), 469-498.

75. G. N. Watson, A new proof of the Rogers-Ramanujan identities, J. London Math. Soc. 4, 4-9.

76. S. O. Warnaar, Hall-Littlewood functions and the $A_{2}$ Rogers-Ramanujan identities, Adv. in Math. 200 (2006), 403-434.

77. S. O. Warnaar and W. Zudilin, Dedekind's $\eta$-function and Rogers-Ramanujan identities, Bull. Lond. Math. Soc. 44 (2012), 1-11.

78. G. N. Watson, Theorems stated by Ramanujan (VII): Theorems on continued fractions, J. London Math. Soc. 4 (1929), 39-48.

79. G. N. Watson, Theorems stated by Ramanujan (IX): Two continued fractions, J. London Math. Soc. 4 (1929), 231-237.

Department of Mathematics, Princeton University, Princeton, NJ 08544, USA

E-mail address: mjg4@princeton.edu 
Department of Mathematics and Computer Science, Emory University, Atlanta, GeorGIA 30322, USA

E-mail address: ono@mathcs.emory.edu

School of Mathematics and Physics, The University of Queensland, Brisbane, AusTRALIA

E-mail address: o.warnaar@maths.uq.edu.au 